\title{
Suppression of coherent synchrotron radiation induced emittance growth during electron-beam injection into plasma wakefields
}

\author{
S.-Y. Kim $\odot$ and M. Chung $\oplus^{*}$ \\ Department of Physics, Ulsan National Institute of Science and Technology, \\ Ulsan 44919, Republic of Korea \\ S. Doebert院 \\ BE Department, CERN, Geneva 23, CH-1211, Switzerland \\ E. S. Yoon $\odot$ \\ Department of Nuclear Engineering, Ulsan National Institute of Science and Technology, \\ Ulsan 44919, Republic of Korea
}

(Received 18 September 2020; accepted 21 January 2021; published 17 February 2021)

\begin{abstract}
Coherent synchrotron radiation (CSR) is a collective effect that mainly occurs when the trajectory of an electron beam is bent in a dipole magnet. It affects the electron beam by distorting the phase space along its slice distribution, which leads to emittance growth. Therefore, CSR should be suppressed to transport electron beams without further degradation of the emittance. In linear optics, CSR-induced emittance can be suppressed by controlling the Twiss parameters along the electron-beam transfer line. However, owing to some physical constraints, transfer-line optics may be governed by higher-order terms in the transfer map, and the use of a sextupole magnet to suppress these terms would be very challenging for low-energyspread and low-emittance beams. Therefore, without using a sextupole magnet, we estimate the region of the Twiss parameters where the first-order terms are dominant along the transfer line by introducing chromatic amplitude. In this region, we can apply the suppression condition that is valid in a linear matrix system. This minimization of the emittance growth becomes even more important when the electron-beam transfer line is used for external injection into a plasma wakefield because mismatched beam conditions could induce an additional increase in the emittance during the acceleration. In this paper, we discuss a method of emittance-growth minimization driven by the CSR effect along the transfer line, which is particularly used for electron-beam injection into plasma wakefields. In addition, using the particle-in-cell simulation, we investigate the evolution of electron beam parameters during the acceleration through plasma wakefields in the presence of the CSR effect on the electron beam. We confirm that the beam emittance growth is minimized when the CSR effect is properly controlled. Otherwise, it is found that 11\%-32\% emittance growths by the CSR effect along the transfer line lead to additional $20 \%-40 \%$ increase of the maximum slice emittance.
\end{abstract}

DOI: 10.1103/PhysRevAccelBeams.24.021301

\section{INTRODUCTION}

A low-emittance, low-energy-spread, short bunchlength, and high-peak-current electron beam is essential for high-quality x-ray generation $[1,2]$ and future highenergy physics facilities such as electron-positron colliders $[3,4]$. For the manipulation and transport of such a highquality electron beam, bending dipole magnets are widely

\footnotetext{
"mchung@unist.ac.kr
}

Published by the American Physical Society under the terms of the Creative Commons Attribution 4.0 International license. Further distribution of this work must maintain attribution to the author(s) and the published article's title, journal citation, and DOI. used in several stages of the beamline. When the trajectory of the electron beam is bent through a dipole magnet, coherent synchrotron radiation (CSR) is emitted [5], which in turn affects the electron beam. CSR is a collective effect that drives the distortion of the phase space along the beam slice. Therefore, it leads to an increase in the projected emittance and further distortion of the energy distribution. In order to transport the beam without further growth in the emittance and energy change, the CSR effect should be controlled.

In the case of free electron laser (FEL), the CSR effect in a bunch compressor has been actively investigated [6-9]. In particular, a collimator has been used in the bunch compressor to remove the double-horn-shaped energy distribution generated by the CSR effect and to minimize 
the emittance growth, thereby improving the x-ray quality $[10,11]$. Likewise, in the beam-driven plasma wakefield acceleration by using the external injection scheme for the electron beam loading, the suppression of the CSR effect becomes also an important issue to be investigated, since one of the challenging issues of plasma wakefield acceleration research is to preserve the emittance of the accelerated electron beam [12-14]. Therefore, we carried out not only the studies on the suppression of the CSR effect along the electron beam transfer line, but also performed the particle-in-cell (PIC) simulation to verify whether the CSR effect leads to additional increase of emittance and RMS energy spread during acceleration through the plasma wakefield.

The suppression of the CSR effect has been actively studied. First, it was shown that the shielding of the radiation by installing metallic plates inside the dipole magnet or by controlling the longitudinal bunch shape can reduce the emittance growth driven by the CSR effect [15-17]. In addition, both measurements and particle tracking simulations have shown that the emittance growth is well suppressed by controlling the phase advance (optics balance) or by matching the linear Twiss parameters between dipole magnets (envelope matching) [18-25]. In both cases, the CSR effect is considered a linear kick and one of the additional dispersive terms associated with the dipole magnet in the linear transfer matrix system. Thus, prior studies have mainly discussed the transfer-line optics dominated by the first-order transfer matrix only. However, in some cases, the transfer-line optics is governed not only by the first-order transfer matrix, but also by higher-order terms that generally produce nonlinear effects. For example, aberration due to the large $\beta$ function of the Twiss parameters and the energy spread of the beam can contribute to the phase-space evolution. In order to apply the suppression method found in preceding studies for minimizing CSR-induced emittance growth, we need to (i) design a transfer line dominated by the first-order matrix system or (ii) find an optimum operating condition in the presence of higher-order components in the transfer line. In this study, we mainly focus on the latter approach.

An achromatic dog-leg electron-beam transfer line and beam parameters are introduced in Sec. II. In Sec. III, the method to minimize the CSR-induced emittance growth in the linear transfer matrix system is discussed. Since we focus on the suppression of the CSR-induced emittance growth in a higher-order-dominated transfer line, in Sec. IV, we introduce the chromatic amplitude and concatenation of the transfer map to find the source of the emittance growth from both higher-order terms and the CSR effect. We also discuss the CSR-induced emittance growth in rectangular dipole magnets that have edge focusing terms and with various longitudinal beam shapes in addition to the Gaussian shape. In Sec. V, considering various conditions for an electron beam extracted from the achromatic dog-leg transfer line, we present the evolution of the emittance, slice distribution and longitudinal phase space during the acceleration through plasma wakefields in the presence of the CSR effect on the electron beam. Finally, we conclude in Sec. VI that the emittance growth can be minimized once the CSR effect is well suppressed before injection.

\section{ACHROMATIC DOG-LEG EXTERNAL INJECTION INTO A PLASMA WAKEFIELD}

To discuss CSR-induced emittance growth, an achromatic dog-leg electron-beam transfer line is introduced. It consists of two horizontal bending dipole magnets with five quadrupole magnets to make dispersion negligible. Figure 1 shows a schematic view of an achromatic dog-leg transfer line with two plasma sources, which was inspired by the future requirements of the AWAKE experiment $[26,27]$. In the first phase of the AWAKE experiments (RUN 1), it was demonstrated for the first time that the energy gain of an electron beam accelerated through a proton-beam-driven plasma wakefield is up to $2 \mathrm{GeV}$ [28]. For the next AWAKE experiment (RUN 2) [29], the first plasma source is planned to be used only for the seeded self-modulation (SSM) of proton beam [30], and the following plasma source is to be used only for the generation of high-gradient plasma wakefields and electron-beam acceleration. Therefore, two plasma sources are placed, between which the electron-beam transfer line is installed. Note that the transfer line presented in this paper serves the purpose of studying the emittance growth due to CSR and its consequences on further injection into plasma wakefields, but it does not represent the current baseline solution for the RUN 2 experiment. Some physical constraints should be considered in this configuration. First, the distance between two plasma sources should be as short as possible to maintain the accelerating gradient from the proton bunches [31]. Therefore, the angle of the bending dipole magnet cannot be made too small (i.e., less than $10^{\circ}$ ) to reduce the distance. In addition, because the quadrupole magnets are installed along the achromatic dog-leg transfer line, overlap between the first plasma source and quadrupole magnets must be avoided. Moreover, the drift length between the second dipole magnet and the last quadrupole magnet should be sufficiently long. The drift space is marked by a red arrow in Fig. 1.

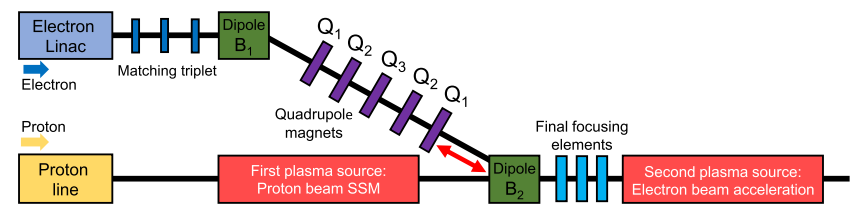

FIG. 1. Schematic view of an electron-beam transfer line with two plasma sources (not to scale). Note that the transfer line in this schematic view is not the AWAKE baseline design but a simplified generic variation to study CSR effects. 
TABLE I. Electron beam parameters.

\begin{tabular}{lc}
\hline \hline Parameter & Value \\
\hline Energy, $E$ & $165 \mathrm{MeV}$ \\
Energy spread, $\sigma_{\delta}$ & $0.1 \%$ \\
Charge, $Q$ & $100 \mathrm{pC}$ \\
Bunch length, $\sigma_{z}$ & $60 \mu \mathrm{m}(200 \mathrm{fs})$ \\
Normalized emittance, $\epsilon_{n}$ & $2 \mathrm{~mm} \mathrm{mrad}$ \\
\hline \hline
\end{tabular}

In this study, it is assumed that the dipole magnet is a sector bending magnet with an arc length of $0.2 \mathrm{~m}$ and a bending angle of $10^{\circ}$. The magnetic length of the quadrupole magnet is assumed to be $0.2 \mathrm{~m}$. The distance between the second dipole magnet and adjacent quadrupole magnet is set to $2.5 \mathrm{~m}$. The electron beam parameters are listed in Table I. The beam energy used for the simulation is $165 \mathrm{MeV}$. The nominal beam charge is $0.1 \mathrm{nC}$, and the root mean square (RMS) bunch length is $60 \mu \mathrm{m}$. The RMS energy spread is assumed to be $0.1 \%$ without chirp, and the beam has the longitudinal shape of a Gaussian distribution with a cutoff boundary of $3 \sigma_{z}$. The normalized emittance is assumed to be $2 \mathrm{~mm}$ mrad. Concerning the Twiss parameters before entering the achromatic dog-leg, they are considered as variables because the suppression condition of the CSR-induced emittance growth should first be found by controlling the Twiss parameters.

Figure 2 shows the Twiss $\beta$ and dispersion functions along the transfer line, with the initial Twiss $\beta_{x}$ function of $0.1 \mathrm{~m}$ and $\alpha_{x}$ function of 0 at the center of the first dipole magnet. The dispersion function becomes zero after the transfer line, which is the main characteristic of the achromat. The transfer-line optics is made symmetric about the center of the third quadrupole magnet to easily satisfy the achromatic condition.

The next task is to find the optimum optics condition for suppressing the CSR-induced emittance growth. Although we eventually aim to minimize the emittance growth in higher-order terms dominated beamline, we start the discussion with the first-order optics system for a comparative study.

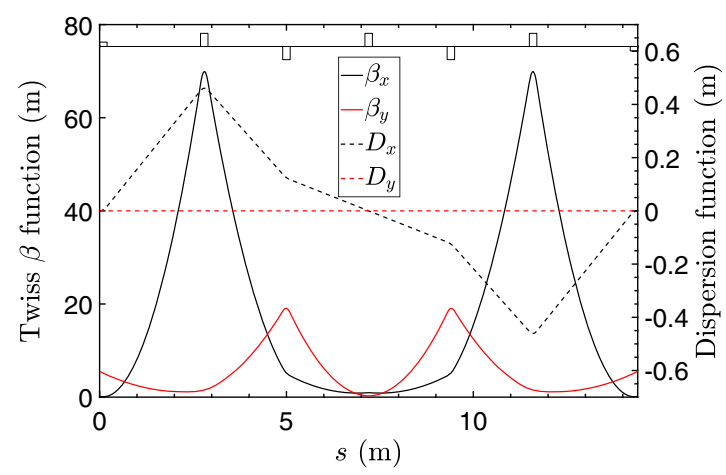

FIG. 2. Twiss $\beta$ and dispersion functions along the transfer line.

\section{SUPPRESSION CONDITION WITH THE FIRST-ORDER TRANSFER MATRIX}

In a linear matrix system, the emittance remains at its initial value if the dispersion is completely suppressed. In this system, a possible source of emittance growth is the collective effect, which is the CSR effect. Following Ref. [23], with envelope matching to minimize the emittance growth, the CSR-induced emittance growth in the linear system is described as

$$
\epsilon_{x, f}=\sqrt{\epsilon_{x, 0}^{2}+\epsilon_{x, 0} d \epsilon_{x}}
$$

where $\epsilon_{x, 0}$ in Eq. (1) is the initial geometrical emittance and $d \epsilon_{x}$ is the fractional growth of the geometrical emittance driven by the CSR effect. If the Twiss parameters satisfy the following condition, $d \epsilon_{x}$ becomes zero:

$$
\left(\frac{2 \alpha_{x}}{\beta_{x}}\right)=\frac{2 \theta}{\rho\left(-2+\theta \frac{C_{1}}{S_{1}}\right)},
$$

where $\beta_{x}$ and $\alpha_{x}$ are the horizontal Twiss parameters at the center of each dipole magnet, respectively; $\theta$ is the bending angle; $\rho$ is the bending radius; $C_{1}$ is $\cos \left(\frac{\theta}{2}\right)$; and $S_{1}$ is $\sin \left(\frac{\theta}{2}\right)$. It is assumed that the initial Twiss $\beta_{x}$ functions at the center of the first (denoted as 1) and second (denoted as 2) dipole magnets are identical $\left(\beta_{x, 1}=\beta_{x, 2}=\beta_{x}\right)$. Similarly, $\alpha_{x, 1}=-\alpha_{x, 2}=\alpha_{x}$, and $\theta_{1}=-\theta_{2}=\theta$. Since the achromatic dog-leg transfer line consists of horizontal bending dipole magnets, CSR mainly affects the horizontal-beam phase space. Therefore, the Twiss parameters in the horizontal plane are controlled to minimize the effect, and $\beta_{x}$ and $\alpha_{x}$ will be denoted as $\beta$ and $\alpha$, unless otherwise indicated. Note that Eq. (2) is obtained using the additional assumption that the CSR kick and the induced RMS energy spread can be calculated using the steady-state mode only. According to Eq. (2) and with the given parameters of the dipole magnet mentioned in Section II, the required Twiss $\alpha$ is approximately -3 if the initial Twiss $\beta$ function is $0.1 \mathrm{~m}$. This condition has been studied using ELEGANT code [32], which supports 1D CSR calculations [7] based on the equations reported in [5]. In addition to the steady-state CSR effect, the present study considers the transient effect at the entrance of the dipole magnet, and radiation effect occurring when the beam propagates to the drift space immediately after the dipole magnet.

In this case, only the first-order matrix system has been used in the ELEGANT tracking simulation. The simulation result of the CSR-induced emittance growth is shown in Fig. 3. In this parametric scan, the initial Twiss $\beta$ function is fixed to $0.1 \mathrm{~m}$, while the Twiss $\alpha$ function is varied from -4 to 4 . The initial position of the beam propagation is in front of the first dipole magnet, and the final emittance was recorded immediately after the second dipole magnet. 


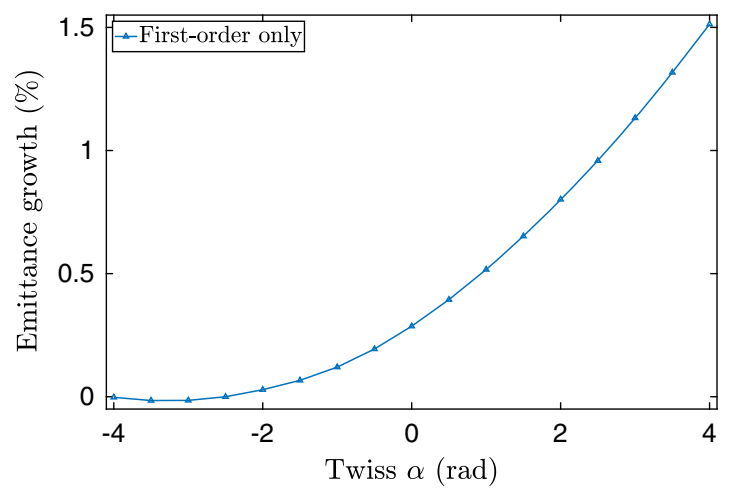

FIG. 3. Emittance growth ratio as a function of initial Twiss $\alpha$ obtained by ELEGANT simulation. In this scan, initial Twiss $\beta$ is fixed to $0.1 \mathrm{~m}$.

As expected from Eq. (2), the emittance growth is minimized when initial Twiss $\alpha \sim-3$.

The emittance growth was also scanned by changing the initial Twiss $\beta$ function together with the initial Twiss $\alpha$ function, as illustrated in Fig. 4. The contour plot shows the growth ratio obtained by the ELEGANT tracking simulation, whereas the lines indicate the ratios calculated by the linear suppression condition. In this Twiss $\alpha$ range, it is confirmed through Fig. 4 that the evaluated ratios using the theoretical model with only the linearized steady-state CSR mode is almost identical to the tracking result where all the CSR effects are considered. For the given dipole magnet parameters such as the arc length of $0.2 \mathrm{~m}$ and bending angle of 10 degrees, the required Twiss $\alpha$ is $-29.985 \beta$ which can be estimated by Eq. (2). Therefore, once the initial Twiss $\beta$ function is increased, the initial Twiss $\alpha$ function should also be increased to suppress the emittance growth caused by the CSR effect. If the initial Twiss $\beta$ function is set to $5.0 \mathrm{~m}$, the required Twiss $\alpha$ function is -149.925 . Once the initial Twiss $\alpha$ function satisfies the linear suppression condition, the emittance growth is perfectly zero. Here, only the linearized steady-state mode of the CSR effect is

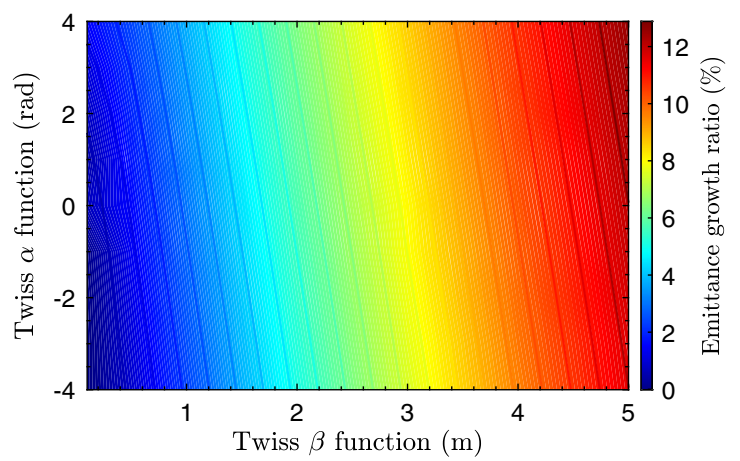

FIG. 4. Emittance growth ratio for a wide range of initial Twiss $\beta$ and $\alpha$ obtained by ELEGANT tracking simulation. Solid lines indicate the ratios calculated by the linear suppression condition obtained from Eqs. (1), (2) [23].
TABLE II. Final normalized emittance from the achromatic dog-leg transfer line. Before entering the dog-leg transfer line, the initial beam emittance was $1.936 \mathrm{~mm}$ mrad.

\begin{tabular}{lllllll}
\hline \hline Twiss $\beta(\mathrm{m})$ & 0.1 & 0.1 & 0.1 & 0.1 & 3.0 & 5.0 \\
Twiss $\alpha(\mathrm{rad})$ & -3.0 & 0.0 & 1.0 & 4.0 & 0.0 & 0.0 \\
\hline Final $\epsilon_{n x}(\mathrm{~mm} \mathrm{mrad})$ & 1.936 & 1.942 & 1.947 & 1.966 & 2.093 & 2.170 \\
Growth ratio $(\%)$ & 0.00 & 0.31 & 0.57 & 1.55 & 8.11 & 12.09 \\
\hline \hline
\end{tabular}

considered. On the other hand, the ELEGANT tracking simulation indicates that the emittance growth becomes $0.93 \%$. It is due to the additional CSR effects such as the transient effect, non-linear effect of the steady-state mode, and CSR drift. Therefore, in the small Twiss $\alpha$ range as illustrated in Fig. 4, the emittance growth becomes significant as the initial Twiss $\beta$ function increases. Table II lists the normalized emittance from the achromatic dog-leg transfer line with various conditions of the Twiss parameters. Because the initial distribution before entering the transfer line is truncated owing to the cutoff boundary of $3 \sigma$, the initial emittance is slightly reduced from the nominal value of $2 \mathrm{~mm}$ mrad to $1.936 \mathrm{~mm}$ mrad.

Figure 5 shows the slice distributions of the electron beam for further discussion of the emittance growth due to the CSR effect. The slice distributions are shown for a reference case not including the CSR effect, a case in which the CSR effect is included but fully suppressed, and a case in which the CSR effect is not properly suppressed. Compared to the reference case, one can see that the phase space is aligned with respect to each slice once the CSR effect is fully suppressed. However, a significant distortion of the beam-slice distribution can be observed if the CSR effect is not fully suppressed. Because the degradation of the energy spread due to the CSR kick is inherently nonlinear and related to the longitudinal beam distribution (for details, see Ref. [7]), the slice distribution is nonlinearly distorted, leading to an increase in the area of the projected phase space. Therefore, the suppression of the CSR effect is important to avoid the emittance growth as well as the distortion of the slice distribution.
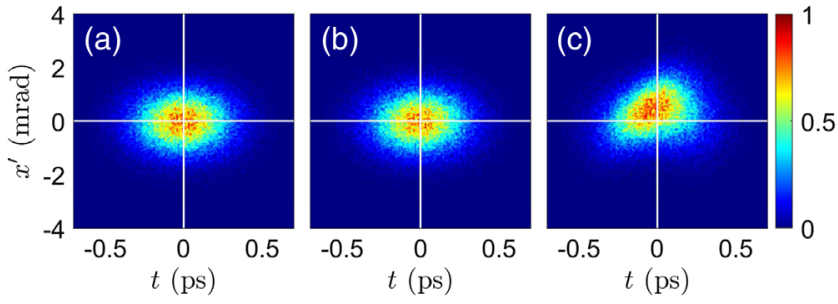

FIG. 5. Beam-slice distribution with various conditions. (a) Reference case in which CSR is not included. (b) CSR is included but fully suppressed by optimizing the Twiss parameters to achieve zero emittance growth. (c) CSR is included and not suppressed, resulting in an emittance growth of $10 \%$. The beam head is placed at negative $t$, and the tail is placed at positive $t$. 


\section{SUPPRESSION CONDITION WITH HIGHER-ORDER TERMS OF THE TRANSFER MAP}

In the previous section, we showed that the CSR-induced emittance growth can be simply minimized by controlling the Twiss parameters in the linear transfer matrix system. However, because the beam has a finite energy spread, chromatic aberration in the quadrupole magnet should also be considered. Moreover, owing to the physical constraints of the transfer line, higher-order terms on the transfer map may play a significant role in the evolution of the beam phase space. One of the ways to eliminate the contribution of the aberration effect is by using a sextupole magnet [33]. However, it introduces additional higher-order terms due to the nonlinearity of the field components, leading to a mismatch of the Twiss parameters along the slice energy distribution. Therefore, this section discusses the minimization of the CSR-induced emittance growth for a case without sextupole magnets. Following each subsection, we investigate the suppression condition when the higher-order terms are dominant along the transfer line.

\section{A. CSR-induced emittance growth with higher-order terms of transfer map}

The transfer map with second-order contributions [34] is expressed as follows:

$$
X_{i}=\sum_{j=1}^{6} R_{i j} X_{j}(0)+\sum_{j=1}^{6} \sum_{k=1}^{6} T_{i j k} X_{j}(0) X_{k}(0), \quad i=1-6,
$$

where $X$ is $\left(x x^{\prime} y y^{\prime} z \delta\right)^{T}, R_{i j}$ is the first-order transfer matrix component, and $T_{i j k}$ is the component of the second-order transfer map. As indicated in Eq. (3), the distortion of the beam phase space and the emittance growth driven by the CSR effect are correlated with the higher-order terms. First, Fig. 6 illustrates the simulation results of the emittance growth due to the CSR effect with consideration of the higher-order terms. In this simulation, all CSR effects were included.

Figure 6(a) shows the relative increase of the normalized emittance obtained using the relation $\left(\epsilon_{\mathrm{ON}}-\epsilon_{\mathrm{OFF}}\right) / \epsilon_{\mathrm{OFF}}$, where $\epsilon_{\mathrm{ON}}$ is the normalized emittance with the CSR effect and $\epsilon_{\mathrm{OFF}}$ is the emittance without the CSR effect. Furthermore, Fig. 6(b) shows the final normalized emittance. Compared to Fig. 4, particularly when the initial Twiss $\beta$ function is $0.1 \mathrm{~m}$, the emittance growth ratio is quite different. On the other hand, as the initial Twiss $\beta$ function increases, the trend of the growth ratio becomes identical to that of the first-order matrix system. Table III summarizes the normalized emittance and its growth ratio for given Twiss parameters. Compared to the values listed in Table II which are obtained with linear matrix system,
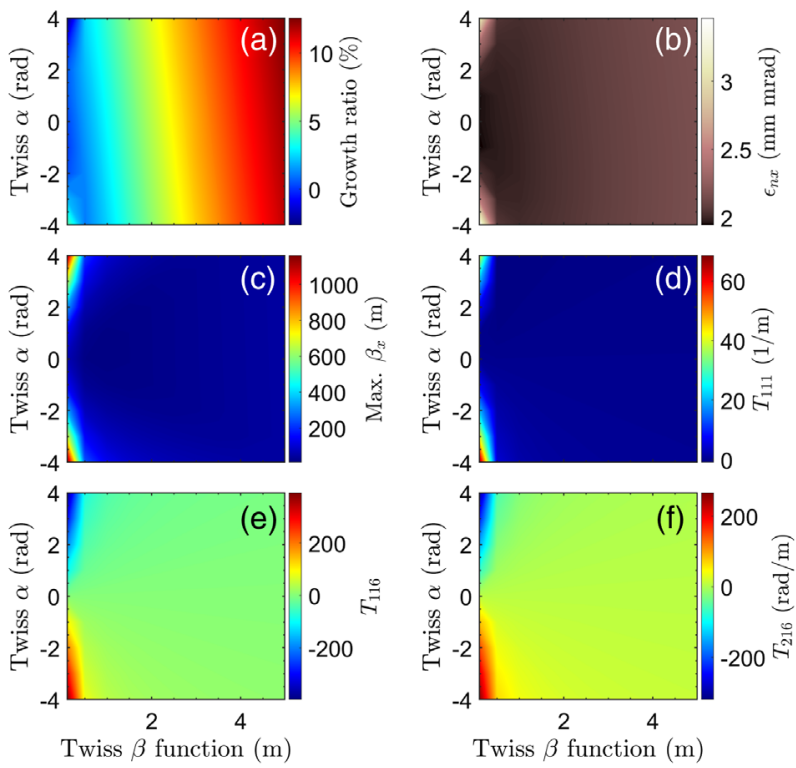

FIG. 6. (a) Relative change of the emittance with higher-order terms considered, (b) final normalized emittance from the achromatic dog-leg transfer line, and (c) maximum Twiss $\beta$ function along the transfer line. Also shown are the second-order transfer map components (d) $T_{111}$, (e) $T_{116}$, and (f) $T_{216}$, with the initial Twiss $\beta$ function ranging from $0.1 \mathrm{~m}$ to $5.0 \mathrm{~m}$ and Twiss $\alpha$ function ranging from -4.0 to +4.0 . All the results in this figure were obtained by ELEGANT simulations.

the absolute values and growth ratios are very different at small Twiss $\beta$ and large absolute values of $\alpha$ functions. On the other hand, at large $\beta$ values, the growth ratios remain similar to the values in Table II. This indicates that for a large initial Twiss $\beta$ function, the transfer line is dominated by the first-order transfer matrix, whereas it is dominated by the higher-order terms when the initial Twiss $\beta$ function is small. It is evident that the higher-order terms play the role of an additional contribution to the degradation of the phase space. This can be confirmed by identifying one of the second-order terms, $T_{111}$, at the end of the transfer line, as shown in Fig. 6(d). In the range where the initial Twiss $\beta$ function is small, $T_{111}$ is large. Thus, the large second-order term leads to a heavy distortion of the phase space. In addition, according to Fig. 6(c), it is clear that the chromatic effect is significant when the maximum Twiss $\beta$ function along the transfer line is large, implying that the beam

TABLE III. Final normalized emittance from the achromatic dog-leg transfer line. Before entering the dog-leg transfer line, the initial beam emittance was $1.936 \mathrm{~mm}$ mrad. Growth ratio was calculated with respect to the initial emittance.

\begin{tabular}{lclllll}
\hline \hline Twiss $\beta(\mathrm{m})$ & 0.1 & 0.1 & 0.1 & 0.1 & 3.0 & 5.0 \\
Twiss $\alpha(\mathrm{rad})$ & -3.0 & 0.0 & 1.0 & 4.0 & 0.0 & 0.0 \\
\hline Final $\epsilon_{n x}(\mathrm{~mm} \mathrm{mrad})$ & 2.478 & 1.958 & 1.955 & 3.099 & 2.094 & 2.172 \\
Growth ratio (\%) & 28.00 & 1.14 & 0.98 & 60.07 & 8.16 & 12.19 \\
\hline \hline
\end{tabular}


envelope is large. Thus, the deviation of $x$ and $x^{\prime}$ in the quadrupole magnet becomes more significant because of the large beam size and beam energy spread, together with the second-order terms $T_{116}, T_{126}, T_{216}$, and $T_{226}$. In Figs. 6(e, f), $T_{116}$ and $T_{216}$ are illustrated.

One can see from Fig. 6(a) that the relative change in the emittance due to the CSR effect is negative in some specific regions. It is expected that higher-order terms may reduce the CSR effect. Nevertheless, as can be seen from Fig. 6(b), the absolute value of the emittance is already larger than the initial value because of the significant contribution of the higher-order terms. Therefore, for the best optimization of the emittance value, we need to control not only the CSR effect, but also the aberrations from the higher-order terms.

\section{B. Chromatic amplitude for the estimation of emittance growth due to higher-order terms}

In order to optimize the beam emittance with higherorder-dominated transfer-line optics, we further investigate the second-order transfer map. In this case, the CSR effect is not considered to focus on the emittance evolution due to only the higher-order terms. Even though the particle coordinates can be described with second-order map components, the Courant-Snyder invariant cannot be simply applied, because the second-order map is nonlinear. Therefore, to perform the optimization process, we introduce the chromatic amplitude [35,36]:

$$
W=\sqrt{\left(\frac{\partial \alpha}{\partial \delta}-\frac{\alpha}{\beta} \frac{\partial \beta}{\partial \delta}\right)^{2}+\left(\frac{1}{\beta} \frac{\partial \beta}{\partial \delta}\right)^{2}},
$$

where $\delta$ is the fractional energy spread and $\beta$ and $\alpha$ are the linear Twiss parameters. The chromatic amplitude represents the deviation of the Twiss parameters with respect to the slice energy distribution. A large chromatic amplitude indicates a large deviation of the Twiss parameters along the beam slice energy distribution. In this case, the slice distribution becomes misaligned, leading to an increase in the projected emittance. Therefore, the minimization of the chromatic amplitude is essential to suppress the emittance growth.

Figure 7(a) shows the simulation result of the emittance from the achromatic dog-leg transfer line as a function of the initial Twiss $\alpha$ function with a fixed initial Twiss $\beta$ value of $0.1 \mathrm{~m}$. As discussed with Fig. 6, the emittance value is overall increased owing to the higher-order terms. At some initial Twiss $\alpha$ values, however, the minimum emittance is observed. This feature can be verified with the slice distribution, as shown in Fig. 7(b). For instance, when the initial Twiss $\alpha$ function is -1.0 , the slice along the energy distribution is well aligned. For all other values of the initial Twiss $\alpha$ function, the slice deviates significantly. Moreover, this behavior can be confirmed by the chromatic amplitude shown in Fig. 8. In the case where the slice distribution is
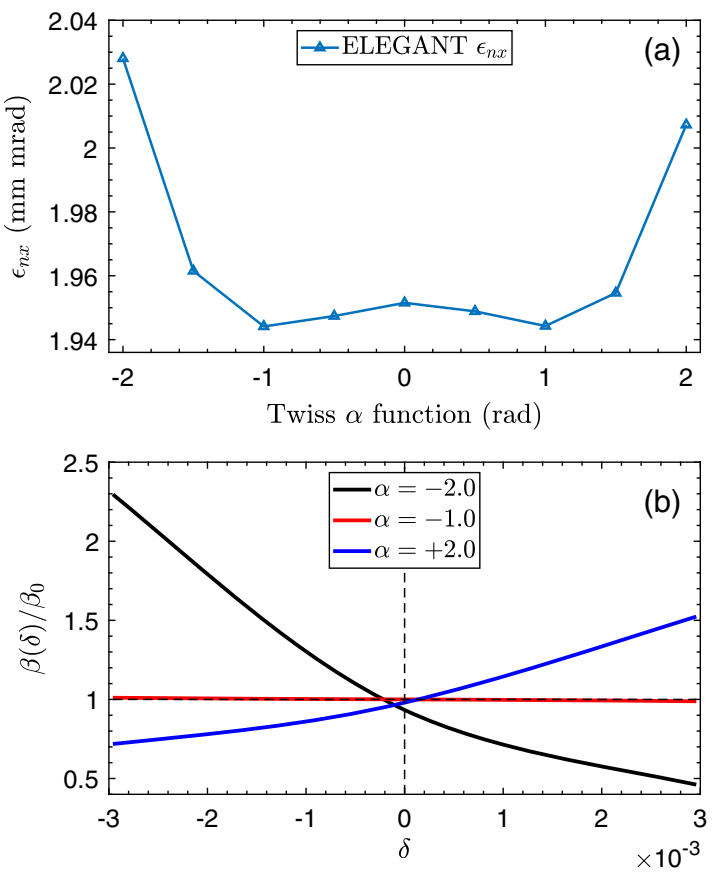

FIG. 7. Normalized emittance from the achromatic dog-leg transfer line (a) and slice Twiss parameters according to the energy distribution (b) obtained using particle tracking. The Twiss initial $\beta$ function is fixed at $0.1 \mathrm{~m}$.

aligned, the chromatic amplitude is minimized. In addition, the variation trend of the chromatic amplitude follows the emittance value in Fig. 7(a). Therefore, in the region where the chromatic amplitude is minimized, the emittance from the achromatic dog-leg transfer line is also minimized. This is important information for the optimization of both the CSR effect and the aberration from the higher-order terms. Furthermore, it can be verified that the evolution of the beam phase space is mainly based on the first-order transfer matrix when the higher-order contribution is minimized.

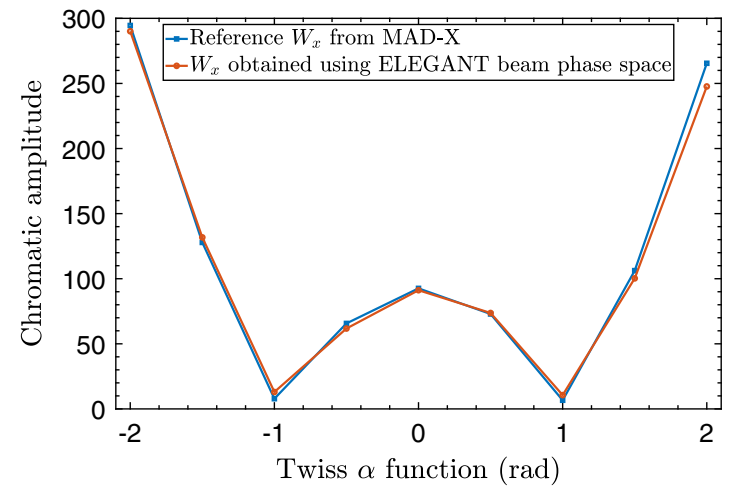

FIG. 8. Chromatic amplitude calculated using Eq. (4) with the phase space obtained from the ELEGANT simulation. The chromatic amplitude evaluated for $\delta=0$ is plotted in this figure. For comparison with the ELEGANT result, the chromatic amplitude was also computed using MAD-X [37] code. 


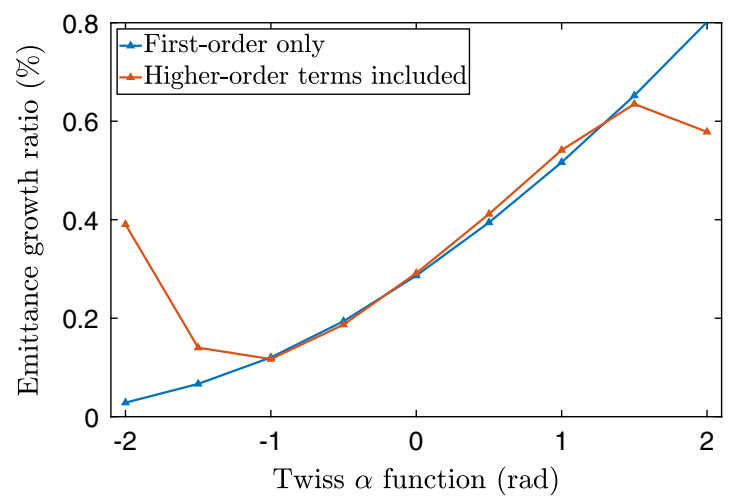

FIG. 9. Emittance growth ratio driven by the CSR effect. The blue curve indicates the ratio with the first-order matrix, whereas the orange curve represents the ratio with the higher-order terms of the transfer map.

\section{Comparison of CSR-induced emittance growths obtained from different models}

Next, we compare the emittance growths driven by the CSR effect in the region where the chromatic amplitude is rather small. In Fig. 9, the emittance growth ratios obtained by the ELEGANT simulation are plotted for the case where only the first-order terms are considered as well as the case where the higher-order terms are considered. In particular, in the region where the chromatic amplitude is minimized (initial Twiss $\alpha$ function ranging from -1.0 to +1.0 ), the trend of the emittance growth in the higher-orderdominated transfer line follows the case with the linear transfer matrix system. Meanwhile, as the chromatic amplitude increases, the growth trend deviates because the higher-order terms are dominant along the transfer line. Moreover, according to Fig. 9, the CSR-induced emittance growth in the higher-order-dominant transfer line is minimized when the initial Twiss $\alpha$ function is -1.0 . At this point, its growth ratio is $0.1 \%$ compared to the emittance in a higher-order system without the CSR effect. With respect to the initial emittance of $1.936 \mathrm{~mm} \mathrm{mrad}$, its final emittance growth ratio is only $0.5 \%$. The optimized growth ratio with considering the higher-order terms of transfer map is slightly larger than the case discussed in Sec. III, where the linear matrix system is considered only. Table IV lists the final emittance value and its growth ratio with several initial Twiss $\alpha$ values.

This condition with the given parameters of the bending dipole magnet is unique. If the initial Twiss $\beta$ function is too small, for instance, $0.05 \mathrm{~m}$, the maximum Twiss $\beta$ function along the transfer line will become extremely large, which can be easily calculated using the transfer matrix that relates the Twiss parameters [38]. This again leads to a huge chromatic aberration. Likewise, when the initial Twiss $\beta$ function is large, the Twiss $\alpha$ function required to compensate for the CSR-induced emittance growth becomes significantly large. This also leads to the
TABLE IV. Final normalized emittance from the achromatic dog-leg transfer line obtained while taking higher-order terms into consideration. Before entering the dog-leg transfer line, the initial beam emittance was $1.936 \mathrm{~mm}$ mrad. The initial Twiss $\beta$ function is fixed at $0.1 \mathrm{~m}$. The emittance growth ratio is estimated relative to the initial emittance.

\begin{tabular}{llll}
\hline \hline Twiss $\alpha(\mathrm{rad})$ & -2.0 & -1.0 & 2.0 \\
\hline Final $\epsilon_{n x}$ without CSR (mm mrad) & 2.028 & 1.944 & 2.007 \\
Final $\epsilon_{n x}$ with CSR $(\mathrm{mm}$ mrad) & 2.036 & 1.946 & 2.018 \\
Growth ratio $(\%)$ & 5.17 & 0.52 & 4.24 \\
\hline \hline
\end{tabular}

aberration due to the higher-order terms and emittance growth. Therefore, to suppress the sources of the emittance growth, such as the aberration from the higher-order terms and the CSR effect, the initial Twiss parameters should be chosen with consideration of both the chromatic amplitude and the suppression condition of the CSR-induced emittance growth.

\section{Suppression condition for the case with a different lattice setting and rectangular dipole magnets}

Also, in order to figure out whether the suppression condition is only determined by given parameters of dipole magnet, we introduced additional quadrupole magnets in between two dipole magnets. Through the simulation, it is also confirmed that the suppression condition of the Twiss parameters is the same for the given parameters of the dipole magnets, even if the number of quadrupole magnets and the lengths of the drift space are different from those in the present setting. This means that the suppression condition is mainly determined by the bending angle and radius when the chromatic amplitude is sufficiently small such that the first-order terms are dominant along the transfer line.

For example, let us assume that a total of 7 quadrupole magnets are used in the achromatic dog-leg transfer line and that the drift space between a dipole magnet and the adjacent quadrupole magnet is $2.5 \mathrm{~m}$. Rectangular bending magnets with finite edge angles are adopted in this case, whereas other main parameters of the dipole magnets remain the same. Figure 10 shows the Twiss parameters along the transfer line and the emittance growth ratio as a function of the initial Twiss $\alpha$ function while the initial Twiss $\beta$ function is fixed at $0.1 \mathrm{~m}$. Compared to the lattice in Fig. 2, it can be seen that the drift spaces in between quadrupole magnets are slightly changed. According to Fig. 10(b), the variation in the emittance growth driven by the CSR effect is almost identical to that shown in Fig. 9. Even using rectangular bending magnet, the suppression condition of CSR-induced emittance growth remains the same as the original case with sector bending magnet. Details of the suppression condition for CSR-induced emittance growth in the rectangular dipole magnet can be found in Appendix A. Therefore, the suppression 

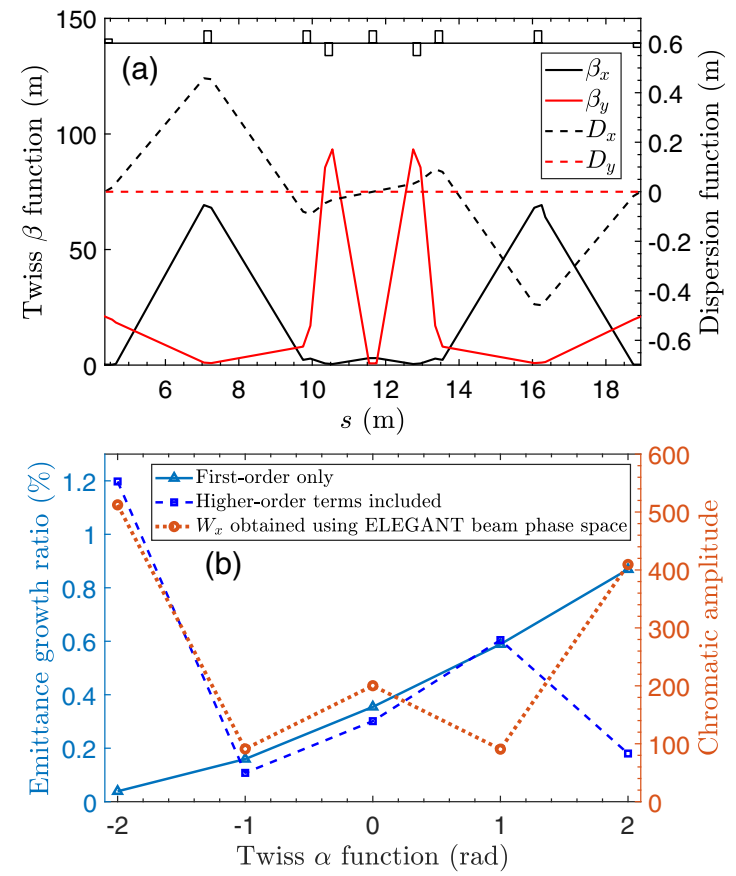

FIG. 10. (a) Twiss parameters and dispersion functions along the achromatic dog-leg transfer line with 7 quadrupole magnets for initial Twiss $\beta=0.1 \mathrm{~m}$ and Twiss $\alpha=0$. (b) Chromatic amplitude and emittance growth ratio.

condition derived in Appendix A is verified by ELEGANT simulation; the edge focusing of rectangular bending magnet does not contribute to determining the suppression condition.

In particular, once the initial Twiss $\alpha$ function becomes +2.0 , the emittance growth due to the CSR effect seems to be decreased. However, owing to the large chromatic amplitude at this value of the initial Twiss $\alpha$ function, the final normalized emittance is $2.193 \mathrm{~mm}$ mrad, indicating that the emittance growth mostly originates from the higher-order terms.

\section{E. Analysis of CSR-induced emittance growth using concatenated matrix}

In addition to the analysis using the chromatic amplitude, we find the source of the emittance growth using the concatenation of the transfer map [39]. For instance, the beam phase space at the end of the first dipole magnet $\left(X_{2}\right)$

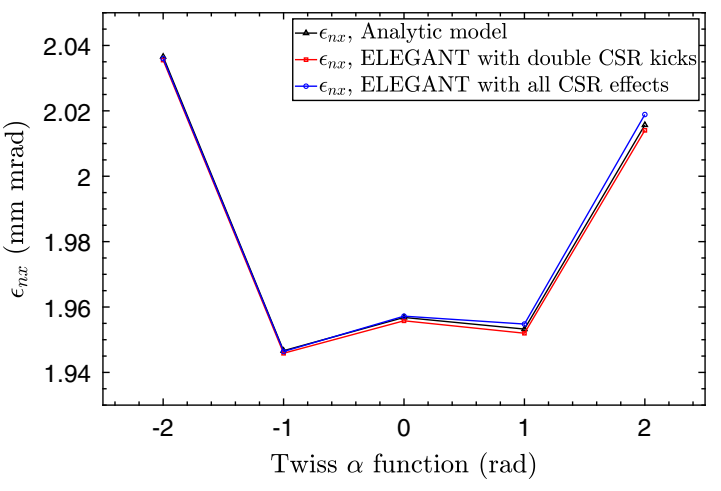

FIG. 12. Emittance variation as a function of the initial Twiss $\alpha$ function. Here, emittance is the value after the achromatic dog-leg transfer line with consideration of both higher-order terms and the CSR effect.

from the initial beam information $\left(X_{0}\right)$, as indicated in Fig. 11, is described by:

$$
\begin{gathered}
X_{1}=M_{B_{11}}\left(X_{0}\right), \\
X_{2}=M_{B_{12}}\left(X_{1}\right)=M_{B_{12}}\left[M_{B_{11}}\left(X_{0}\right)\right],
\end{gathered}
$$

where $M_{B_{11}}$ and $M_{B_{12}}$ are the components of the dipole magnet's transfer map including the second-order components. Following the concatenation in Eq. (6), the achromatic dog-leg lattice is set up as illustrated in Fig. 11. Here, $\delta_{0}$ is the fractional energy spread of the initial beam distribution, and $\delta_{C S R}$ is the CSR-induced energy change. To apply the CSR kick, a bending dipole magnet is separated by two slices. Once the beam passes through the first slice of each dipole magnet, a single CSR kick is applied. Subsequently, the energy change due to the CSR kick is updated. In this calculation, it is assumed that the bunch length remains constant along the transfer line and the distribution does not change, indicating that the CSR kick is identical for all slices of the dipole magnet. In addition, the CSR kick is based on the steady-state mode with a Gaussian beam shape. Based on these assumptions, we set up a model to find the source of the emittance growth in the presence of the CSR effect as well as the higher-order components.

Figure 12 shows the normalized emittance from the achromatic dog-leg transfer line obtained from the

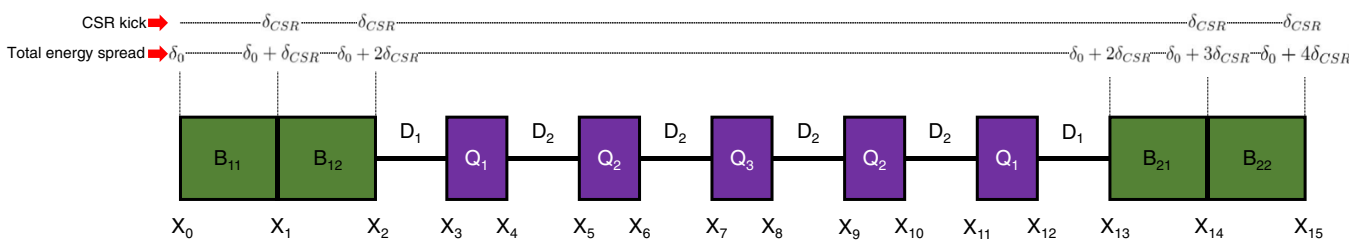

FIG. 11. Achromatic dog-leg transfer line lattice for calculating the emittance growth with both the higher-order terms and CSR effect. $D_{1}$ and $D_{2}$ are the elements of the drift space, and $Q_{1}, Q_{2}$, and $Q_{3}$ are the quadrupole magnets. $B_{11}$ and $B_{12}$ are slices of the first sector bending dipole magnet, and $B_{21}$ and $B_{22}$ are slices of the second sector bending dipole magnet. 
analytical model and the ELEGANT simulation. In these calculations, the initial Twiss $\beta$ function was fixed at $0.1 \mathrm{~m}$, and the initial $\alpha$ function was varied from -2.0 to +2.0 . In order to verify the validity of the analytical model, the ELEGANT tracking is performed with the following assumptions: (i) double kicks with the steady-state mode of the CSR effect and (ii) multiple kicks with all the CSR effects (transient, steady-state, and CSR drift). Compared to the two cases of the simulation results, a small discrepancy in the analytical model can be observed. This is mainly due to the following assumptions in the analytical model: a constant bunch length along the entire transfer line and an invariant longitudinal distribution. Nevertheless, the variation of the emittance with higher-order terms and the CSR effect can be estimated using the analytical model to a good approximation.

The following equations describe the deviations of the final beam phase space $\Delta x_{15}$ and $\Delta x_{15}$ 'in Fig. 11 due to only the CSR effect $\delta_{\mathrm{CSR}}$ :

$$
\begin{aligned}
\Delta x_{15}= & M_{15,0001} \delta_{\mathrm{CSR}}+M_{15,1001} x_{0} \delta_{\mathrm{CSR}}+M_{15,0101} x_{0}{ }^{\prime} \delta_{\mathrm{CSR}}+M_{15,0011} \delta_{0} \delta_{\mathrm{CSR}}+M_{15,0002} \delta_{\mathrm{CSR}}^{2}+M_{15,2001} x_{0}{ }^{2} \delta_{\mathrm{CSR}} \\
& +M_{15,1101} x_{0} x_{0}{ }^{\prime} \delta_{\mathrm{CSR}}+M_{15,1011} x_{0} \delta_{0} \delta_{\mathrm{CSR}}+M_{15,1002} x_{0} \delta_{\mathrm{CSR}}{ }^{2}+M_{15,0201} x_{0}{ }^{2} \delta_{\mathrm{CSR}}+M_{15,0111} x_{0}{ }^{\prime} \delta_{0} \delta_{\mathrm{CSR}} \\
& +M_{15,0102} x_{0}{ }^{\prime} \delta_{\mathrm{CSR}}{ }^{2}+M_{15,0021} \delta_{0}^{2} \delta_{\mathrm{CSR}}+M_{15,0012} \delta_{0} \delta_{\mathrm{CSR}}^{2}+M_{15,0003} \delta_{\mathrm{CSR}}^{3},
\end{aligned}
$$

$$
\begin{aligned}
\Delta x_{15}{ }^{\prime}= & M_{15,0001}^{\prime} \delta_{\mathrm{CSR}}+M_{15,1001}^{\prime} x_{0} \delta_{\mathrm{CSR}}+M_{15,0101}^{\prime} x_{0}{ }^{\prime} \delta_{\mathrm{CSR}}+M_{15,0011}^{\prime} \delta_{0} \delta_{\mathrm{CSR}}+M_{15,0002}^{\prime} \delta_{\mathrm{CSR}}^{2}+M_{15,2001}^{\prime} x_{0}{ }^{2} \delta_{\mathrm{CSR}} \\
& +M_{15,1101}^{\prime} x_{0} x_{0}{ }^{\prime} \delta_{\mathrm{CSR}}+M_{15,1011}^{\prime} x_{0} \delta_{0} \delta_{\mathrm{CSR}}+M_{15,1002}^{\prime} x_{0} \delta_{\mathrm{CSR}}{ }^{2}+M_{15,0201}^{\prime} x_{0}{ }^{\prime 2} \delta_{\mathrm{CSR}}+M_{15,0111}^{\prime} x_{0}{ }^{\prime} \delta_{0} \delta_{\mathrm{CSR}} \\
& +M_{15,0102}^{\prime} x_{0}{ }^{\prime} \delta_{\mathrm{CSR}}{ }^{2}+M_{15,0021}^{\prime} \delta_{0}^{2} \delta_{\mathrm{CSR}}+M_{15,0012}^{\prime} \delta_{0} \delta_{\mathrm{CSR}}^{2}+M_{15,0003}^{\prime} \delta_{\mathrm{CSR}}^{3},
\end{aligned}
$$

where terms such as $M_{15,0001}$ and $M_{15,0001}^{\prime}$ are the concatenated components, and $x_{0}, x_{0}{ }^{\prime}$, and $\delta_{0}$ are phase-space information of the initial particle before entering the achromatic dog-leg transfer line. In the concatenated matrix $M_{n, i j k l}, n$ indicates the sequence number in the transfer line, and indexes $i, j, k$, and $l$ are associated with the integer power terms correspond to $x_{0}^{i}, x_{0}^{\prime j}, \delta_{0}^{k}$, and $\delta_{\mathrm{CSR}}^{l}$, respectively. Although terms of order higher than three occur in this calculation, they can be ignored because they do not significantly affect the results. In addition, terms related to the vertical components $y_{0}$ and $y_{0}{ }^{\prime}$ can be ignored because these terms are negligibly small. Because each transfer-map component is a combination of the first- and second-order maps, it is extremely tedious to list all the components here. As examples for the combination of the map components, $M_{15,0001}$ and $M_{15,0001}^{\prime}$ are listed in Appendix B.

When only the first-order matrix is considered, the modulation of the phase space due to the CSR effect is expressed by only a single term related to $M_{15,0001}$ and $M_{15,0001}^{\prime}$ in Eqs. (7), (8). However, if higher-order terms are considered, many additional terms are generated. The final geometrical emittance can then be expressed as follows:

$$
\epsilon_{x}=\sqrt{\left\langle\left(x_{15}+\Delta x_{15}\right)^{2}\right\rangle\left\langle\left(x_{15}{ }^{\prime}+\Delta x_{15}{ }^{\prime}\right)^{2}\right\rangle-\left\langle\left(x_{15}+\Delta x_{15}\right)\left(x_{15}{ }^{\prime}+\Delta x_{15}{ }^{\prime}\right)\right\rangle^{2}},
$$

where $x_{15}$ and $x_{15}{ }^{\prime}$ are the final phase-space coordinates without the CSR effect.

Because $\delta_{\mathrm{CSR}}$ is correlated with the transverse phase space, it cannot be easily separated in Eq. (9) to obtain a simple relation for the suppression condition. Therefore, using Eqs. (7), (8), we show the beam phase space distorted only by the CSR effect. Figure 13 shows the phase-space distortion driven by the terms related to the $\delta_{C S R}$ estimated using Eqs. (7), (8). As indicated in Fig. 13, the phase space is greatly distorted when the initial Twiss $\alpha$ function is \pm 2.0 (cases 1 and 2), where the emittance growth ratio is rather large. Once the emittance growth is minimized (case 3), the area of the phase-space evolution driven by the CSR effect is minimized.

\section{F. Suppression condition with different longitudinal beam shapes}

In the previous discussions on the suppression condition for CSR-induced emittance growth, it was always assumed that the longitudinal beam shape follows a Gaussian distribution. However, for realistic cases, the longitudinal profile may not always be Gaussian; it can be, for instance, a flat-top or parabolic profile. Therefore, if we consider the 


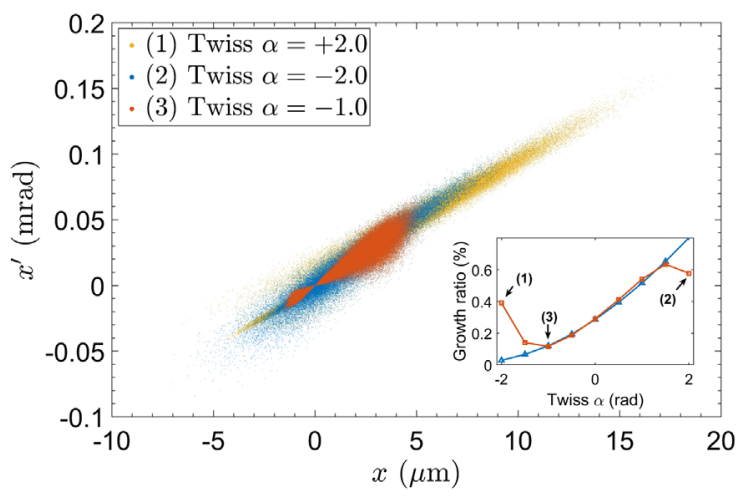

FIG. 13. Distorted phase space resulting from the CSR effect estimated using Eqs. (7), (8).

steady-state mode of the CSR effect, the energy change induced by CSR will be different from the case of an ideal Gaussian shape. This energy change can be calculated as follows [7]:

$$
\frac{d E(s)}{c d t}=\int_{-\infty}^{s} \frac{d \lambda}{d z}\left(\frac{1}{s-z}\right)^{1 / 3} d z
$$

where $\lambda$ is the line charge distribution of the beam. Figure 14 shows the CSR-induced energy changes in various longitudinal beam distributions extracted using the ELEGANT simulation.

In order to determine how to find the optimum suppression condition, the CSR-induced RMS energy spread was calculated using the ELEGANT simulation, as illustrated in Fig. 15. In this simulation, a single sector bending dipole magnet was used, and all CSR effects were considered. The initial RMS length, energy spread, and beam charge are set to the same values as those indicated in Sec. II. For the Gaussian distribution, the energy spread $\Delta \sigma_{\delta}$ is proportional to $\rho^{1 / 3} \theta$, as discussed in Ref. [23]. In the case of a flat-top or parabolic distribution, it can be deduced from

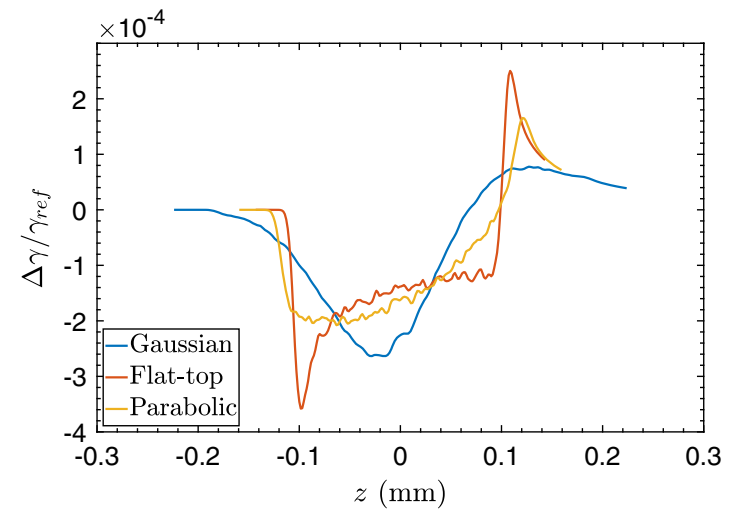

FIG. 14. Energy change induced by the CSR effect with several beam distributions. Only the steady-state mode was turned on. The beam head is located at positive $z$, whereas the tail is at negative $z$.

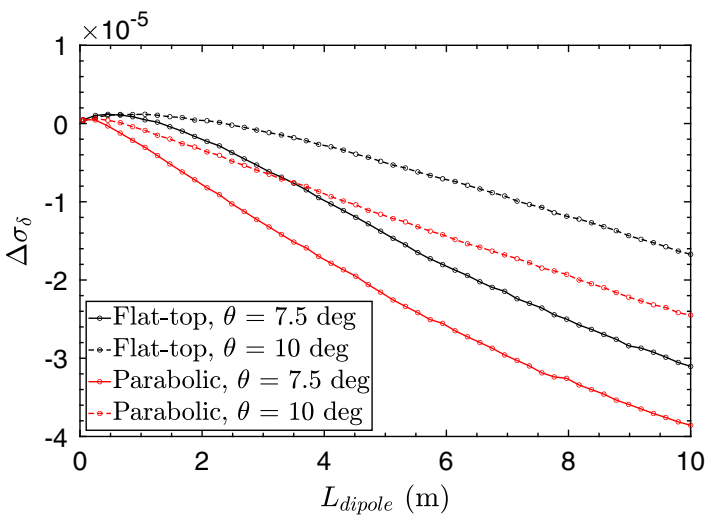

FIG. 15. RMS energy spread induced by the CSR effect as a function of the arc length $L_{\text {dipole }}$ of the dipole magnet. Here, $\Delta \sigma_{\delta}=\sigma_{\delta, \mathrm{ON}}-\sigma_{\delta, \mathrm{OFF}}$, where $\sigma_{\delta, \mathrm{ON}}$ is the RMS energy spread with the CSR effect and $\sigma_{\delta, \mathrm{OFF}}$ is that without the CSR effect.

Fig. 15 that $\Delta \sigma_{\delta} \propto-L_{\text {dipole }}=-\rho \theta$, except for the regime in which the length of the dipole magnet is rather short. In particular, the equation of motion with the CSR effect in the bend plane is made linear with this assumption, which allows us to evaluate the suppression condition in the linear system. By adopting this assumption, the equations of motion and linear transfer matrix components are modified from those indicated in Ref. [20]. The equation of motion in the bend plane is then given by

$$
x^{\prime \prime}=-\frac{x}{\rho^{2}}+\frac{1}{\rho}\left[\delta_{0}+\delta_{\mathrm{CSR}}-k\left(s-s_{0}\right)\right],
$$

where $s_{0}=0$ is the entrance of the dipole magnet. Here, $k$ is normalized CSR wake potential that depends on the bunch length and charge. Then, the coordinate deviations due to the CSR kick become

$X_{e}=\left(\begin{array}{c}x_{e} \\ x_{e}^{\prime}\end{array}\right)=\left(\begin{array}{c}\rho(1-\cos \theta) \\ \sin \theta\end{array}\right) \delta+\left(\begin{array}{c}-\rho^{2}(\theta-\sin \theta) \\ -\rho(1-\cos \theta)\end{array}\right) k$,

where the sign in the second term is different from that in Eq. (A1), which can be found by Green's function method [40]. Then, the point-kick model is given by

$$
X_{k}=\left(\begin{array}{c}
x_{k} \\
x_{k}^{\prime}
\end{array}\right)=\left(\begin{array}{c}
\rho^{2} k\left(-\theta \cos \frac{\theta}{2}+2 \sin \frac{\theta}{2}\right) \\
\sin \frac{\theta}{2}(2 \delta-\rho \theta k)
\end{array}\right) \text {. }
$$

Following the sequence to find the suppression condition, as indicated in Sec. III of Ref. [23] with $\delta=-k \rho_{1} \theta_{1}$, we can find the optimum Twiss parameters for the minimization of the CSR-induced emittance growth, which is given by 


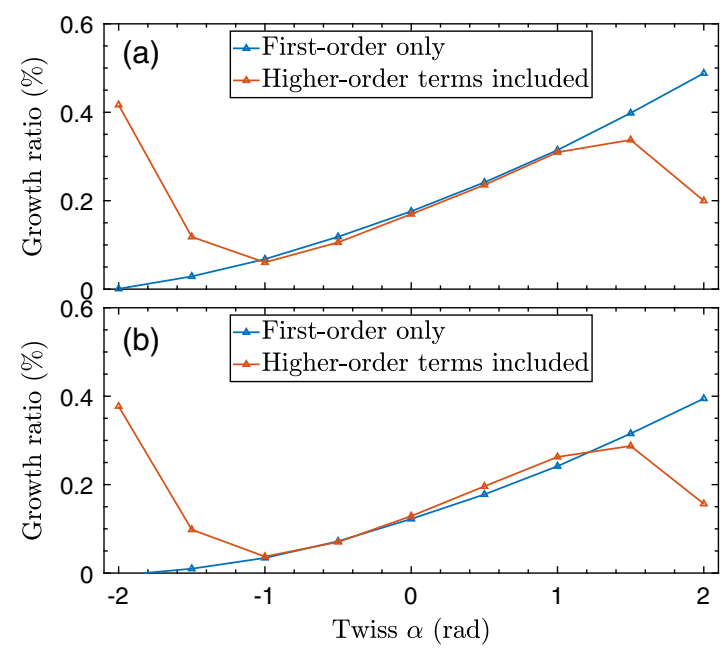

FIG. 16. Emittance growth ratio due to the CSR effect for (a) a flat-top distribution and (b) a parabolic distribution. The blue curve indicates the ratio estimated with the first-order matrix. The orange curve is the ratio calculated by including the higher-order terms of the transfer map.

$$
\left(\frac{\alpha_{1}-\alpha_{2}}{\beta_{1}}\right)=\frac{\left(\theta_{1}+r^{*} \theta_{2}\right)}{\rho_{1}\left(-2+\theta_{1} \frac{C_{1}}{S_{1}}\right)},
$$

where $r^{*}$ is $\rho_{2} / \rho_{1}$. Interestingly, even if the change in the RMS energy spread is different for the specific longitudinal beam shape, the suppression condition remains the same form as that obtained in the case of a Gaussian distribution.

To verify our assumption, the ELEGANT simulation was performed again using the achromatic dog-leg transfer line. In this simulation, all CSR effects were included. Figure 16 shows the emittance growth ratios of different longitudinal beam distributions. Compared to Fig. 9, the evolution trend of the emittance growth is very similar to that of the Gaussian beam case, even though the longitudinal beam profile has changed. Furthermore, the CSR-induced emittance growth is minimized at the initial Twiss $\alpha$ of -1.0 when the higher-order contributions are considered (the orange curve in Fig. 16). This indicates that our assumption is valid, and the suppression condition is the same for several different beam distributions.

\section{EMITTANCE VARIATION DURING ACCELERATION THROUGH A PLASMA WAKEFIELD}

In this section, we discuss the variation of the electronbeam emittance during the acceleration inside a plasma wakefield in the presence of the CSR effect. As indicated in Fig. 1, the electron beam passing through the achromatic dog-leg transfer line is focused by the final focusing element and propagates to the second plasma source. To investigate the emittance variation due to only the CSR effect during the beam loading scenario, a first-order matrix system was used for particle tracking along the achromatic dog-leg transfer line. Furthermore, it is assumed that the beam envelope is matched by using permanent-magnet quadrupoles (PMQs) [41] at the final focusing section. In the matching of the beam envelope, the betatron oscillation inside the blowout regime is minimized when the beam envelope is set by the following condition [42]:

$$
\sigma_{r m}=\left(\frac{2 \epsilon_{0} \gamma m_{e} c^{2} \epsilon_{g}^{2}}{e^{2} n_{e}}\right)^{1 / 4}
$$

where $\epsilon_{0}$ is the vacuum permittivity, $\gamma$ is the relativistic factor of the electron beam, $m_{e}$ is the electron mass, $c$ is the speed of light, $\epsilon_{g}$ is the geometrical emittance, $e$ is the elementary charge, and $n_{e}$ is the plasma density. In the case of the AWAKE experiment, the plasma density of the acceleration column is $7 \times 10^{4} / \mathrm{cm}^{3}$. With the given electron beam parameters listed in Table I, the required RMS beam size at the entrance of the plasma source is approximately $5.6 \mu \mathrm{m}$. Following Ref. [43] for the beam loading scheme, we performed the particle-in-cell (PIC) simulation using a single proton bunch. Particle tracking inside the plasma wakefield was performed using Fourier-Bessel PIC (FBPIC) simulation code [44]. The number of grid cells was set to 1190 in the transverse plane and 2735 in the longitudinal plane in a cylindrical simulation box, where the radius of the transverse box is $1 \mathrm{~mm}$ and the longitudinal size is $2.3 \mathrm{~mm}$, respectively. The resolution of the simulation is approximately $0.84 \mu \mathrm{m}$ in both the transverse and longitudinal planes. The simulation was performed in the boosted frame with the parameter $\gamma_{\text {boosted }}=32$ to reduce the simulation time. The number of azimuthal modes used in the simulation is up to 3 . The plasma density distribution and the length of the plasma source were set to be uniform without density ramp and $5.0 \mathrm{~m}$, respectively.

Figure 17 shows the plasma wakefield, driving proton beam, witness electron beam, and plasma density modulation. For this simulation, the mass of the proton particle is increased by 6 orders of magnitude to sustain the wake amplitude over a long distance. The RMS beam size $\sigma_{r}$ is $200 \mu \mathrm{m}$, the RMS bunch length is set to $40 \mu \mathrm{m}$, and the energy of the proton beam is $400 \mathrm{GeV}$. The charge of the proton beam used for this simulation is $2.34 \mathrm{nC}$. The particle data obtained using the ELEGANT simulation are used for the witness beam. The number of macroparticles of the proton and electron beams is $2.1 \times 10^{6}$ and $1.0 \times 10^{6}$, respectively. The position of the electron beam was chosen such that the effective RMS energy spread and normalized emittance are minimized. Figure 18 shows longitudinal phase space of the electron beam after propagation through $1 \mathrm{~m}$ plasma source. Here, the initial electron beam center is located behind the proton beam center at a distance of $1.21 \mathrm{~mm}$. When the total number of electron beam particles is considered, the RMS energy spread becomes $4.00 \%$. As 


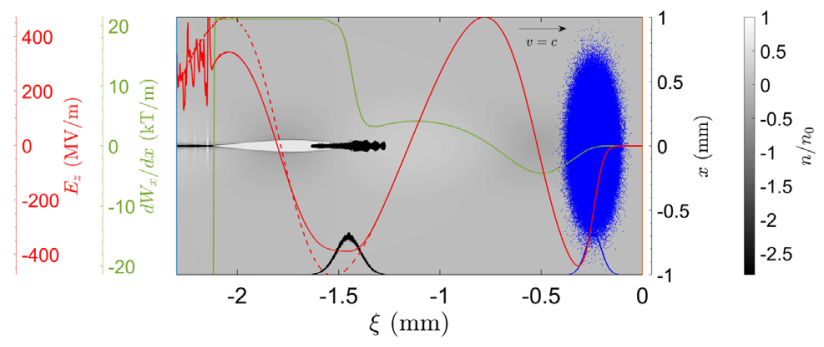

FIG. 17. Plasma wakefield driven by a single proton bunch (blue dots). The electron beam (black dots) is injected to be accelerated with a strong focusing gradient. The solid red plot indicates the longitudinal plasma wakefield with a witness electron beam, while the dashed red line represents the wakefield created only by the proton beam. The transverse focusing field $W_{x}=E_{x}-c B_{y}$ and the focusing gradient (green line) were evaluated near the axis $(x \sim 0)$. The longitudinal coordinate $\xi=z-c t$. Here, $n / n_{0}>0\left(n / n_{0}<0\right)$ means there are more positively (negatively) charged species.

can be seen in Fig. 18(a), large RMS energy spread is mainly due to the energy spread at the electron beam head region where the amplitude of the plasma wakefield is not constant. However, in the shaded region around the $\delta$ peak as depicted in Fig. 18(b), the effective RMS energy spread (calculated with the particles in the region where $\delta \geq 0$ and the density in arbitrary unit is larger than 0.001) becomes $0.85 \%$, and the acceleration efficiency (the number of electron beam particles in the shaded region) becomes approximately $76 \%$. Using the Gaussian fitting, the RMS energy spread inside the narrow region is calculated to be $0.31 \%$. Simultaneously, the normalized emittance is $2.532 \mathrm{~mm}$ mrad.

In Fig. 17, the maximum longitudinal wake amplitude induced by the electron beam is around $380 \mathrm{MV} / \mathrm{m}$.

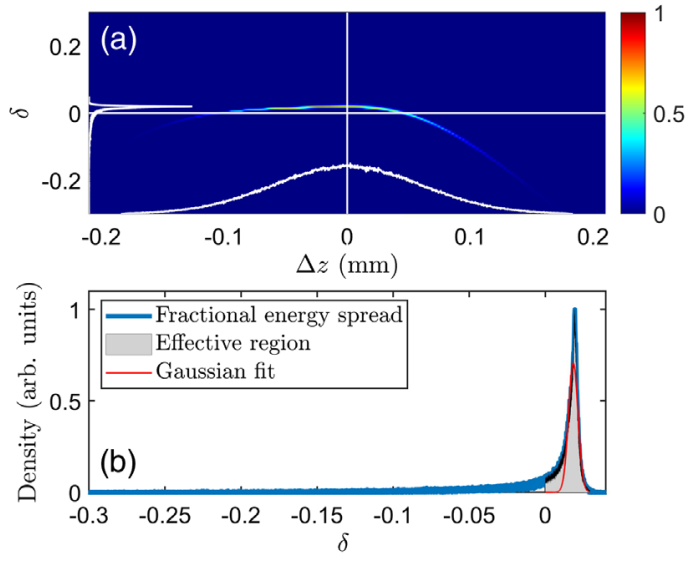

FIG. 18. (a) Longitudinal phase space of the electron beam after propagation through $1 \mathrm{~m}$ plasma source. Electron beam head is placed at positive $\Delta z$, whereas the tail is located at negative $\Delta z$. (b) Fractional energy spread distribution. Shaded area is considered to calculate effective RMS energy spread. Red line is the Gaussian fit curve.
Because the witness electron beam has a Gaussian distribution, the longitudinal wakefield cannot be perfectly flat to induce a constant energy gain in the electron beam. In addition, the electron beam density is approximately 31 times larger than the ambient plasma density; consequently, the witness beam creates the blowout regime. In this regime, the focusing gradient is constant, and its strength is nearly $20 \mathrm{kT} / \mathrm{m}$. However, because the head part of the beam is not in the blowout regime, a different focusing gradient is applied along the slice. Therefore, the head of the electron beam is rather defocused compared to the rest of the beam, which is in the blowout regime, leading to head erosion $[45,46]$.

In order to study the emittance variation of the electron beam with the CSR effect, several cases of the electron beam were used for the PIC simulation. In the linear matrix system, we consider (i) a reference case without the CSR effect, (ii) a case in which the CSR effect is fully suppressed so that the emittance growth is $0 \%$ in the transfer line, and (iii) a case in which the CSR effect is not suppressed, leading to emittance growths of $11 \%, 21 \%$, and $32 \%$ after the dog-leg transfer line. The phase space of the electron beam before entering the plasma source is shown in Fig. 19. It can be seen that the CSR effect nonlinearly deviates the electron beam phase space. Once the CSR effect is minimized, the transverse and slice distributions in Figs. 19 (b, g) are almost identical to the reference case in Figs. 19 (a, f). However, if the CSR effect is not suppressed, the beam centroid is largely shifted and the slice distribution is significantly distorted, as illustrated in Figs. 19 (c-e) and (h-j).

By using the beam phase space shown in Fig. 19, a beam loading simulation was conducted. First, the characteristics of the electron-beam phase space in the reference case are discussed. The slice distribution of the electron beam after propagation through the $1 \mathrm{~m}$ plasma source is shown in Fig. 20(a). In Figs. 20(b) to (d), the horizontal phase space is plotted at the tail, center, and head of the beam, respectively. The normalized emittance in the horizontal plane after the plasma source is $2.532 \mathrm{~mm}$ mrad, which is somewhat increased from the initial value of $1.936 \mathrm{~mm}$ mrad. This emittance growth is mainly due to the deviation of the slice phase space, which results from the different focusing gradients induced to the beam slice, as illustrated in Fig. 17. At the electron beam head, the focusing gradient is smaller than that inside the blowout regime. Thus, the beam envelope at the head is expanded compared to that at the beam tail. In addition to the different focusing gradients along the slice, the gradient outside the blowout regime is not linear with respect to the transverse position, as shown in Fig. 20(e). This is an example of the betatron decoherence $[14,47,48]$ due to the different betatron oscillations arising from different focusing gradients. Furthermore, this betatron decoherence occurs in the same manner because of the finite energy spread of the electron 

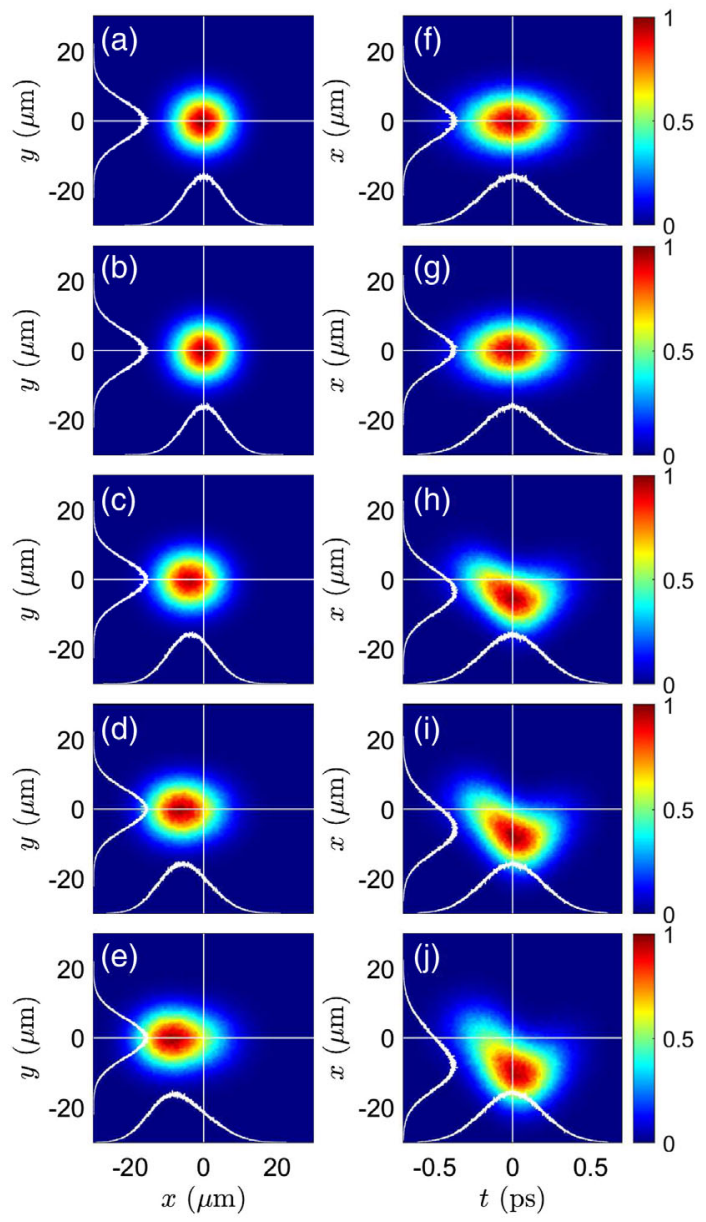

FIG. 19. Electron-beam projections (a-e) and slice distributions $(\mathrm{f}-\mathrm{j})$ before entering the plasma source obtained by ELEGANT simulation. ( $\mathrm{a}, \mathrm{f})$ are for the reference case without CSR effect, $(b, g)$ are for the case in which the CSR effect is fully suppressed so that the emittance growth is nearly $0 \%$, and $(\mathrm{c}, \mathrm{h})$ are for the case in which the CSR effect is not suppressed, resulting in the emittance growth of $11 \%$. Also, $(\mathrm{d}, \mathrm{i})$ are the case where the CSRinduced emittance growth is $21 \%$, and $(e, j)$ are the case where the emittance growth is $32 \%$. The beam head is located at negative $t$, while the beam tail is placed at positive $t$.

beam and the different accelerating gradients along the beam slice, as shown in Fig. 17. The following equation describes the single-particle motion with acceleration and the linear focusing gradient:

$$
\frac{d^{2} x}{d s^{2}}+\frac{\gamma(s)^{\prime}}{\gamma(s)} \frac{d x}{d s}+\frac{1}{\gamma(s)}\left(\frac{d F_{x}}{d x}\right) x=0
$$

where it is assumed that $s \equiv z$, together with the variable $\xi=z-c t . \gamma(s)=\gamma_{0}+E_{z} s, \gamma_{0}$ is the initial relativistic factor of each particle, $E_{z}$ is the longitudinal wakefield normalized by $\frac{e}{m_{e} c^{2}}$, and $F_{x}=\frac{e}{m_{e} c^{2}}\left(E_{x}-c B_{y}\right)$. Thus, the term $\frac{d F_{x}}{d x}$ represents the transverse focusing gradient. $\gamma(s)^{\prime}$ is a derivative with respect to the variable $s$. According to the
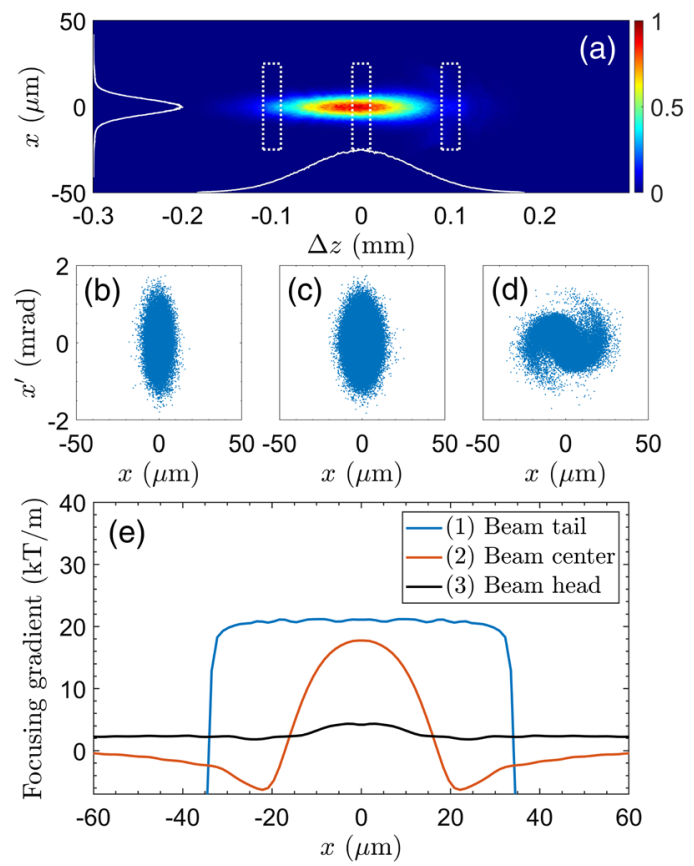

FIG. 20. (a) Slice distribution of the electron beam after propagation through the $1 \mathrm{~m}$ plasma source. The beam head is placed at positive $\Delta z$, while the beam tail is placed at negative $\Delta z$. The dashed boxes indicate the regions of the slice distributions. (b, c, d) Slice horizontal phase space at different slice locations at the tail, center, and head of the beam, respectively. (e) Focusing gradient along three different electron-beam slices.

third term in Eq. (16), the betatron oscillation frequency $K=\sqrt{\frac{1}{\gamma(s)}\left(\frac{d F_{x}}{d x}\right)}$ is not constant along the beam slice at a fixed position of the propagation axis, owing to the different $\gamma_{0}$ and the longitudinal wakefield, in addition to the different focusing gradients along the slice. The inconsistent wake amplitude as well as the finite energy spread lead to additional betatron decoherence, resulting in further emittance growth.

The emittance growth is saturated once the betatron decoherence process is complete, as can be expected from the second term in Eq. (16), which indicates the damping of the betatron oscillation amplitude. By changing the variable $s$ to $\psi=\sqrt{\frac{4}{E_{z}{ }^{2}}\left(\frac{d F_{x}}{d x}\right)} \eta$, where $\eta=\gamma(s)^{1 / 2}$, Eq. (16) is modified to $\frac{d^{2} x}{d \psi^{2}}+\frac{1}{\psi} \frac{d x}{d \psi}+x=0$. Then, the solution of the modified equation of single-particle motion will be [49]

$$
\begin{gathered}
x(\psi)=C_{1} J_{0}(\psi)+C_{2} Y_{0}(\psi), \\
x^{\prime}(\psi)=-C_{1} J_{1}(\psi)-C_{2} Y_{1}(\psi),
\end{gathered}
$$

where $J_{0}, J_{1}, Y_{0}$, and $Y_{1}$ are Bessel functions of the first kind, and $C_{1}$ and $C_{2}$ are coefficients that can be determined by the initial particle conditions. Based on Ref. [49], if the initial transverse divergence $x^{\prime}=0$ and the variable $\psi \gg 1$, 
Eq. (17) becomes $x_{0}\left(\psi_{0} / \psi\right)^{1 / 2} \cos \left(\psi-\psi_{0}\right)$. Thus, the oscillation amplitude is proportional to $\left(\gamma_{0} / \gamma(s)\right)^{1 / 4}$. As the beam energy monotonically increases, the amplitude of the betatron oscillation decreases rapidly, and the emittance becomes saturated once the oscillation amplitude is negligibly small. In the following subsections, using several electron beam with CSR effect, evolution of beam envelope and emittance along the plasma source will be discussed.

\section{A. Reference case and case where the CSR effect is fully suppressed}

Figure 21 plots the evolutions of the RMS beam size and beam centroid, as well as the saturation of the normalized emittance obtained from the simulation. Solid blue line indicates the case without CSR effect (reference case), while the orange line represents the case where the CSR effect is considered, but fully suppressed. Inserts of Fig. 21 are the plots for the colored boxes. For all cases, large oscillation of the beam envelope and emittance growth were observed. The oscillation amplitude of the beam envelope and emittance rapidly decrease at approximately $0.5 \mathrm{~m}$. Up to this region, the betatron decoherence significantly affects the beam emittance, leading to a huge
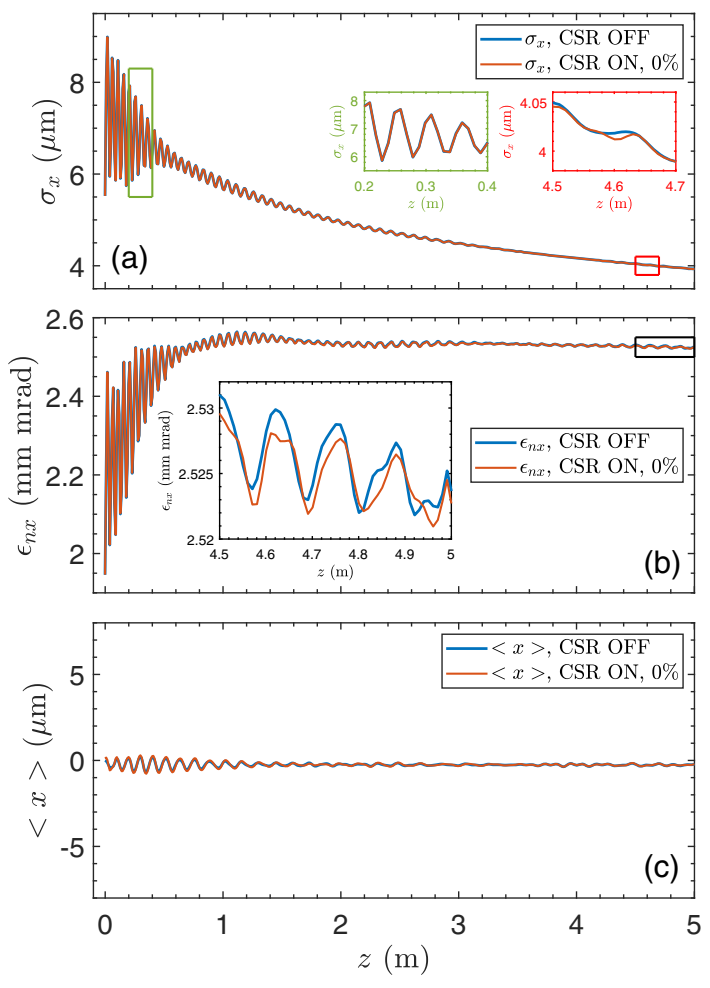

FIG. 21. Plots of (a) RMS beam envelope, (b) normalized emittance, and (c) horizontal beam centroid along the plasma source. CSR OFF is the case in which the CSR effect is not considered along the transfer line, and CSR ON is the case in which the CSR effect is considered. In the figure legends, $0 \%$ represents zero emittance growth driven by the CSR effect. Inserts are zoomed plots for the boxed regions.
TABLE V. Final normalized emittance from the $5 \mathrm{~m}$ plasma source. The ratio was calculated with respect to the initial emittance of $1.936 \mathrm{~mm}$ mrad.

\begin{tabular}{lcc}
\hline \hline & CSR OFF & CSR ON \\
\cline { 2 - 3 } Case & Reference & 0\% growth \\
\hline Final $\epsilon_{n x}(\mathrm{~mm} \mathrm{mrad})$ & 2.524 & 2.523 \\
Growth ratio $(\%)$ & 30.37 & 30.32 \\
\hline \hline
\end{tabular}

emittance growth. In the reference case without CSR effect, the emittance is saturated, and its value increases by nearly $30 \%$ from the initial value of $1.936 \mathrm{~mm}$ mrad after completion of the betatron decoherence. As the beam propagates to the plasma source while gaining energy, the oscillation period gradually increases. In this simulation study, the focusing gradient is sustained over a long distance from the plasma source, and the electron beam energy is gradually increased by the longitudinal wakefield. Thus, the betatron oscillation frequency, which is the coefficient in the third term of Eq. (16), decreases. Consequently, the wavelength corresponding to the oscillation frequency increases as can be seen in inserts of Fig. 21(a).

The normalized emittance after propagation through $5 \mathrm{~m}$ plasma source is summarized in Table V. In the case where the CSR effect is fully suppressed, the emittance is nearly identical to the reference case. Therefore, once the CSR effect along the electron beam transfer line is fully suppressed, the emittance growth after acceleration through the plasma wakefield becomes negligible. Also, in this case, the centroid oscillation after saturation of the betatron decoherence becomes very small as shown in Fig. 21(c).

\section{B. Case where the CSR effect is not suppressed}

Figure 22 shows the case where the CSR effect is not suppressed. In the legends, $11 \%, 21 \%$, and $32 \%$ represent the emittance growth ratios along the transfer line due to the CSR effect. Solid lines indicate the case where the electron beam centroid and angle before entering the plasma source are not adjusted, whereas dashed lines are the cases where the centroid and angle are assumed to be adjusted. First, large oscillation of the beam envelope and centroid position can be seen in Figs. 22(a,c). In the case without adjustment, the emittances as shown in Fig. 22(b) are somewhat increased compared to the values described in Table V. Interestingly, when the centroid and angle are assumed to be adjusted, the final emittances become even larger. Table VI lists the final emittances for these cases. The additional emittance growth driven by the CSR effect can be analyzed in terms of slice emittance. Figure 23 shows the slice emittances after $5 \mathrm{~m}$ plasma source where (a) is the case where the beam centroid and angle are not adjusted and (b) is the case where those are assumed to be adjusted. The reference case and the case where the CSR effect is fully suppressed are plotted as blue and orange lines, 

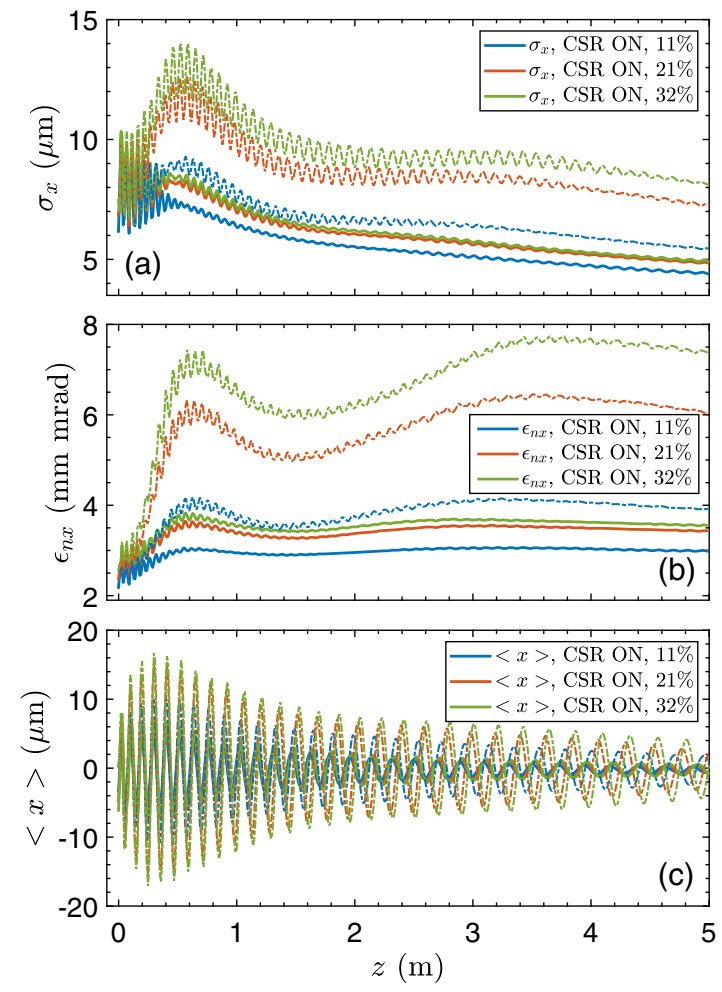

FIG. 22. Plots of (a) RMS beam envelope, (b) normalized emittance, and (c) horizontal beam centroid along the plasma source. In the figure legends, $11 \%, 21 \%$, and $32 \%$ indicate the emittance growth by CSR effect before entering the plasma source from the initial emittance of $1.936 \mathrm{~mm}$ mrad. Solid lines are the case without adjusting the electron beam centroid and angle before injection to the plasma wakefield, whereas the dashed lines are the cases with adjustment.

respectively. In these cases, the emittances are increased mainly at the head of the electron beam. It is due to the head-erosion effect; in the beam head, the transverse focusing field is not constant along the slice nor linear in the transverse direction, leading to huge phase-space distortion [see Fig. 20(d)]. Meanwhile, the emittance inside the blow-out regime remains almost the same as the initial emittance of $1.936 \mathrm{~mm}$ mrad. However, when the CSR effect is imprinted in the beam, we observe a noticeable growth of the head-erosion effect as shown in Fig. 23(a).

TABLE VI. Final normalized emittance from the $5 \mathrm{~m}$ plasma source. CSR $11 \%, 21 \%$, and $32 \%$ indicate that the CSR-induced emittance growths before entering the plasma source are $11 \%$, $21 \%$, and $32 \%$ from the initial emittance of $1.936 \mathrm{~mm} \mathrm{mrad}$.

\begin{tabular}{lccc}
\hline \hline & CSR & CSR & CSR \\
Case & $11 \%$ & $21 \%$ & $32 \%$ \\
\hline $\begin{array}{l}\text { With centroid and angle not adjusted, } \\
\quad \text { Final } \epsilon_{n x} \text { (mm mrad) }\end{array}$ & & & \\
$\begin{array}{l}\text { With centroid and angle adjusted, } \\
\text { Final } \epsilon_{n x}(\mathrm{~mm} \mathrm{mrad})\end{array}$ & 3.911 & 6.041 & 7.337 \\
\hline \hline
\end{tabular}
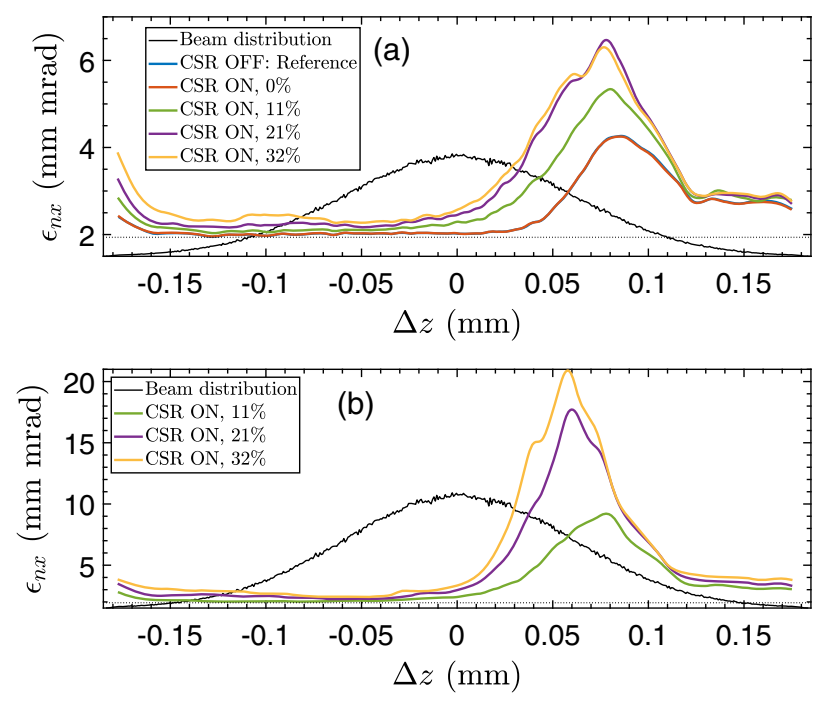

FIG. 23. Slice emittance along the electron beam after propagation through $5 \mathrm{~m}$ plasma source. (a) Cases where the beam centroid and angle are not adjusted. (b) Cases where those are adjusted. Black solid and dashed lines indicate the longitudinal electron beam distribution in arbitrary units and initial emittance of $1.936 \mathrm{~mm}$ mrad, respectively. Electron beam head is placed at positive $\Delta z$, whereas the tail at negative $\Delta z$.

There are additional $20-40 \%$ increases in the maximum slice emittance. It is due to not only the misalignment of the beam centroid and angle with respect to the center of the plasma source [50], but also the mismatch of the slice distribution as shown in Fig. 19.

Furthermore, Fig 23(b) shows that the head-erosion effect becomes even severer with the adjustment. In particular, in case of Fig. 19(j), the beam core in the horizontal position is negatively shifted. When the centroid position is adjusted to make $\langle x\rangle$ zero, the overall distribution will be positively shifted, leading to the large misalignment at the head part of the electron beam. Consequently, there are additional 110-310\% increases in the maximum slice emittance. Table VII summarizes the enhancement of the head-erosion effect due to the CSR.

By observing the slice emittance, we found that due to the CSR effect emittance is additionally increased at the electron beam head region. It is mainly due to the nonlinear distortion of the beam phase space. In terms of the

TABLE VII. Enhancement of the head-erosion effect due to the CSR calculated using the maximum emittance along the beam slice.

\begin{tabular}{llll}
\hline \hline & CSR & CSR & CSR \\
Case & $11 \%$ & $21 \%$ & $32 \%$ \\
\hline $\begin{array}{l}\text { With centroid and angle not adjusted, } \\
\quad 22.24\end{array}$ & 36.43 & 22.70 \\
$\quad \begin{array}{lll}\text { Additional growth ratio (\%) } \\
\text { With centroid and angle adjusted, } \\
\quad \text { Additional growth ratio (\%) }\end{array}$ & 110.61 & 273.51 & 306.26 \\
\hline \hline
\end{tabular}


projected emittance as shown in Fig. 22, the additional emittance growth due to the CSR effect is approximately $10 \%$ when the centroid and angle of the electron beam are not adjusted. In case where those are adjusted, the additional emittance growth becomes as high as $120 \%$. Therefore, the adjustment of the centroid and angle of the beam in presence of the CSR effect hinders the improvement of the beam parameters during the acceleration through plasma wakefields.

\section{Electron beam slice distribution and longitudinal phase space after plasma source}

Figure. 24 illustrates the slice distributions (first row), longitudinal phase spaces (second row) and fractional energy distributions (third row) after propagation through $5 \mathrm{~m}$ plasma source. In particular, Fig. 24 shows the cases where the centroid and angle of the electron beam are not adjusted before entering the plasma source. The slice distribution of the reference case without CSR effect can be seen in Fig. 24(a), whereas the case where the CSR effect is considered but fully suppressed is illustrated in Fig. 24(d). The slice distribution in which the CSR is fully suppressed is very similar to the reference case. However, if the CSR-induced emittance growth is not properly suppressed and this electron beam is injected into the plasma source, additional distortion at the beam head are observed as illustrated in Figs. 24(g, j, m). In addition, microbunchinglike instability appears to start at the beam tail.

Regarding the longitudinal phase space in Figs. 24(b, e, $\mathrm{h}, \mathrm{k}, \mathrm{n}$ ), it is observed that there is no significant change.
Since we mainly focus on CSR-induced emittance growth, we may expect that the energy distribution of the electron beam is also somewhat distorted by the CSR effect. Nevertheless, from the ELEGANT tracking simulation with 1D CSR approximation [7], the deviation of the RMS energy spread due to the CSR effect is less than $0.005 \%$ which is negligibly small. Therefore, it is expected that the effective RMS energy spread during acceleration through the plasma source is mainly determined by the longitudinal component of the wakefield. For the comparative analysis on the energy spread, three different methods to calculate the RMS energy spread have been used. Firstly, we calculated the RMS energy spread by considering the entire electron beam particles. Secondly, the effective RMS energy spread has been calculated by considering the region where the fractional energy spread $\delta$ is larger than 0 and the density value (arb. units) is larger than 0.001 . Lastly, standard deviation $\sigma_{\delta}$ has been found using the Gaussian fitting over the effective regions as can be seen in Figs. 24 (c, f, i, 1, o). The RMS energy spreads obtained by these three different methods are summarized in Table VIII as well as average energy. For each calculation method, it is verified that the calculated RMS energy spread values are similar to the reference case. When the total number of electron beam particles is considered, the RMS energy spread is approximately $5.5 \%$ because the amplitudes of the longitudinal wakefield at the head and tail regions are not constant and largely deviated as shown in Fig. 17. It is significantly larger than that in a conventional accelerator which is in the order of $0.1 \%$. However, when considering
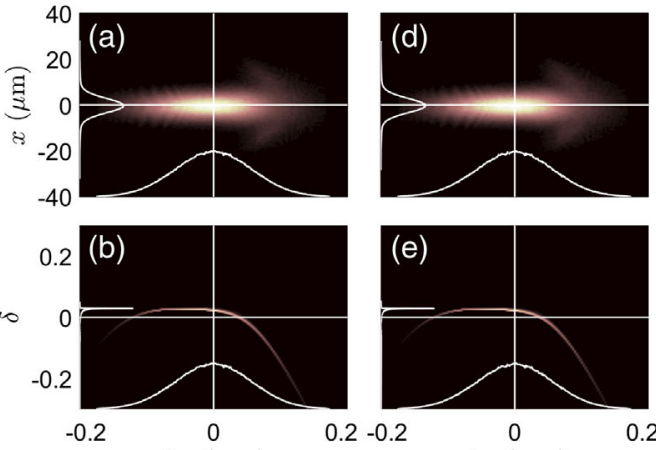

$0.2-0.2$
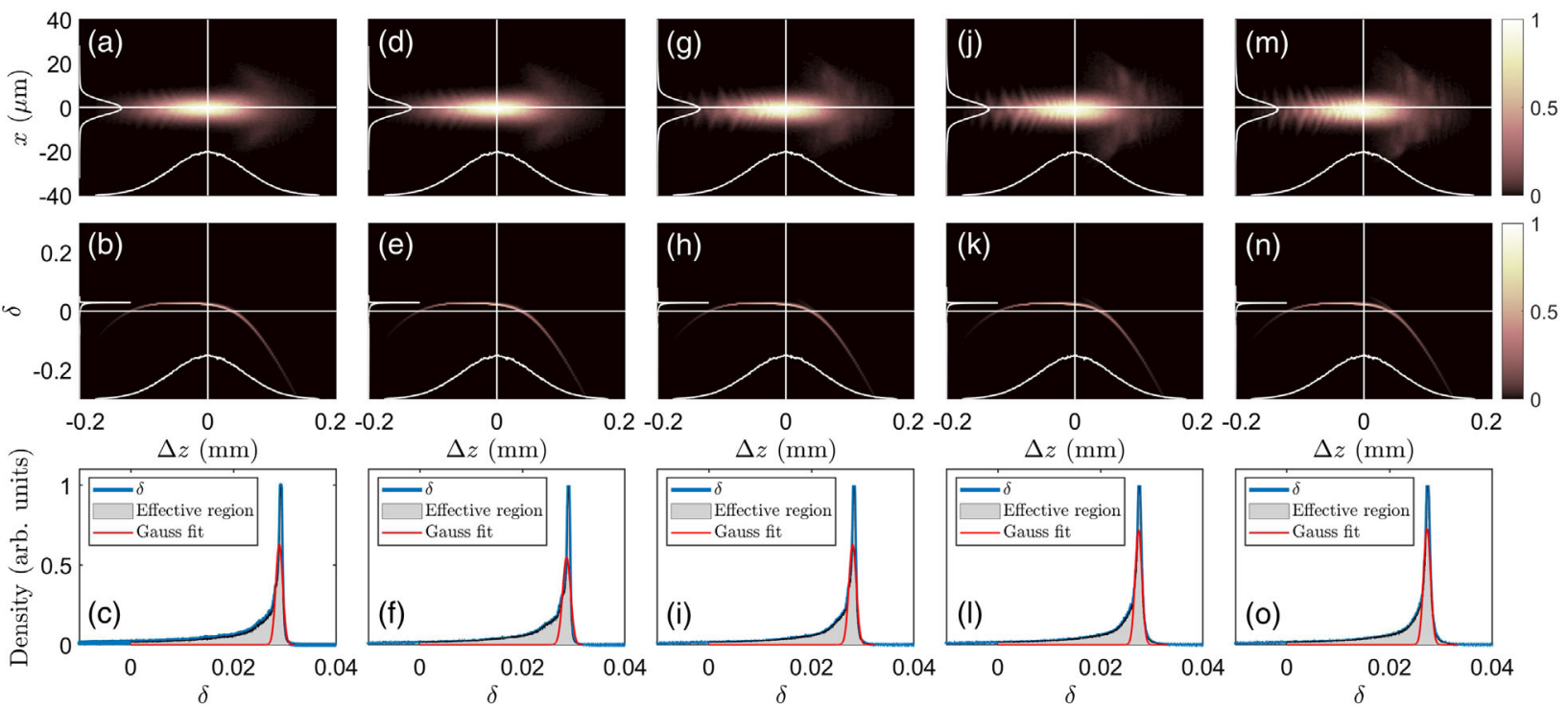

FIG. 24. Beam slice distribution (first row), longitudinal phase space (second row), and fractional energy spread (third row) of the electron beam after propagation through the $5 \mathrm{~m}$ plasma source under various conditions. (a-c) Reference case with CSR OFF. (d-f) Case in which CSR is ON but fully suppressed, resulting in zero emittance growth. (g-i) Case in which CSR is ON and not suppressed, resulting in an emittance growth of $11 \%$. (j-1) Case where the CSR-induced emittance growth is $21 \%$. (m-o) Case where the CSR-induced emittance growth is $32 \%$. For the cases where the CSR effect is not suppressed, the centroid and angle are not adjusted. The beam head is placed at positive $\Delta z$ and the tail at negative $\Delta z$. 
TABLE VIII. Average energy $\left(E_{\text {avg }}\right)$ and RMS energy spread of the electron beam after propagation through $5 \mathrm{~m}$ plasma source. Methods a, b, and c are the cases where the RMS energy spreads obtained by (a) considering total electron beam particles, (b) considering effective region (shaded area in the third row of Fig. 24), and (c) using Gaussian fitting over the effective region.

\begin{tabular}{lccccc}
\hline \hline Case & Reference & $\begin{array}{c}\text { CSR } \\
0 \%\end{array}$ & $\begin{array}{c}\text { CSR } \\
11 \%\end{array}$ & $\begin{array}{c}\text { CSR } \\
21 \%\end{array}$ & $\begin{array}{c}\text { CSR } \\
32 \%\end{array}$ \\
\hline$E_{\text {avg }}(\mathrm{GeV})$ & 2.016 & 2.016 & 2.017 & 2.019 & 2.019 \\
Method a (\%) & 5.548 & 5.509 & 5.523 & 5.542 & 5.553 \\
Method b (\%) & 0.923 & 0.918 & 0.936 & 0.957 & 0.959 \\
Method c (\%) & 0.068 & 0.078 & 0.072 & 0.066 & 0.069 \\
\hline \hline
\end{tabular}

only the effective region as depicted in Figs. 24(c, f, i, 1, o), it is confirmed that in all cases, the RMS energy spread is reduced to less than $1 \%$ and the acceleration efficiency becomes approximately $75 \%$. In addition, the RMS energy spread obtained by using Gaussian fitting remains similar within the error range of $\pm 0.0047 \%$. As a consequence, it has been found that the small amount of CSR-induced energy spread along the electron beam does not affect the energy spread after propagation through the plasma wakefield.

By noting that a low energy spread and high quality elecron beam can be maintained in the FEL by removing double-horn shape in the energy distribution [10], we may further assume that the electron beam particles are collimated to remove the head and tail regions where the energy spreads are larger. Then, approximately $25 \%$ of the electron beam particles are cut out by the collimator, and the remaining electron beam has the effective RMS energy spread of nearly $0.9 \%$. Simultaneously, by cutting off the head part of the electron beam where the head-erosion effect is dominant, it is possible to keep the final emittance as close as the initial value before entering the plasma source. Nevertheless, still the emittance inside the effective region would be increased by the existing CSR effect on the electron beam as shown in Fig. 23(a). (e.g., see the emittance in the region from $\Delta z=-0.15 \mathrm{~mm}$ to $0.04 \mathrm{~mm}$ ). Therefore, in order to avoid emittance growth, suppression of the CSR effect along the transfer line should be performed. By controlling the CSR effect to make the zero emittance growth along the transfer line, the emittance at the effective region can almost be maintained by the initial value of $1.936 \mathrm{~mm}$ mrad.

\section{CONCLUSION}

The suppression of the emittance growth driven by the CSR effect along the electron-beam transfer line was discussed for efficient beam loading into a plasma wakefield accelerator. In the linear transfer line optics system, the CSR-induced emittance growth can be suppressed by controlling the Twiss parameters between the dipole magnets of the achromatic dog-leg transfer line. When the higher-order terms in the transfer map play significant roles in the phase-space evolution, we need to consider those terms for the suppression of the CSR-induced emittance growth. This condition can be determined by estimating the chromatic amplitude. In the regime where the chromatic amplitude is small, it was confirmed that the transfer map is dominated by the first-order matrix. In this case, we can apply the suppression condition that is valid in the linear transfer matrix system. Finally, we found that the emittance growth can be minimized within a ratio of $0.5 \%$ compared to the initial emittance value by simultaneously controlling the Twiss parameters to make the chromatic amplitude small.

This minimization is crucial for electron-beam loading in a plasma wakefield because the ultimate goal of the plasmawakefield acceleration is to reduce emittance growth and make it applicable to high-energy physics experiments and high-quality $\mathrm{x}$-ray generations. In the case where the CSR-induced emittance is fully suppressed before the beam loading, PIC simulation verified that the emittance growth is almost identical to that in the reference case without the CSR effect. Otherwise, the emittance growth becomes more significant. As discussed previously, emittance growths of $11 \%, 21 \%$, and $32 \%$ along the transfer line lead to additional enhancement of the head-erosion effect by 20\% 40\% after propagation through the plasma wakefield. Also, the additional emittance growth ratio in terms of projected emittance is around $10 \%$. The main factor of the emittance growth driven by the CSR effect is due to the slice mismatch at the beam head with respect to the entire electron beam slice. Therefore, when the electron beam is externally injected for plasma wakefield acceleration, it is important to control the CSR effect to minimize the emittance degradation. In terms of the energy spread, since the CSR-induced energy change of the electron beam along the achromatic dog-leg is not significant, the RMS energy spread remains similar regardless of whether the CSR effect is fully suppressed, or it is not suppressed.

Furthermore, if the effective region of electron beam is only considered for practical application, it is expected that the emittance growth and RMS energy spread can be simultaneously controlled by cutting off the electron beam's head and tail regions. By suppressing the CSR effect along the electron beam transfer line together with the collimation method, it would be possible to keep the emittance growth as minimal as possible. In this context, it is of critical importance to control the CSR effect in the external injection scheme for the electron beam loading into the plasma wakefields.

\section{ACKNOWLEDGMENTS}

This work was supported by the National Research Foundation (NRF) of Korea (Grant No. NRF-2016Global Ph.D. Fellowship Program and No. NRF2020R1A2C1010835). This research was also supported by 


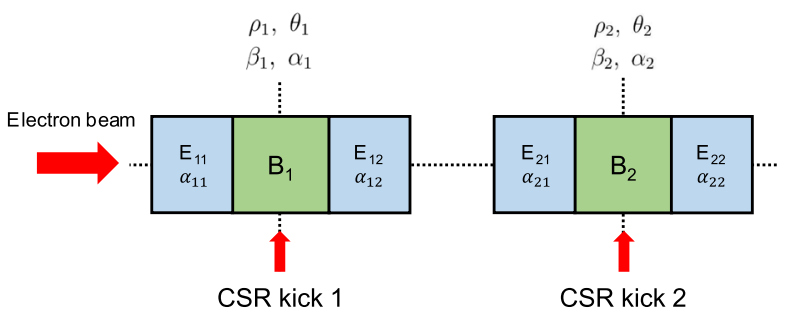

FIG. 25. Schematic view of the achromatic dog-leg transfer line with edge angles.

R\&D program (code No. IN2004-6) through the Korea Institute of Fusion Energy funded by the Government funds.

\section{APPENDIX A: CSR SUPPRESSION CONDITION IN RECTANGULAR BENDING MAGNET}

Equation (2) was obtained using the sector bending dipole magnet. If it is replaced by a rectangular bending magnet, the transfer matrix for edge focusing should be considered. Following Sec. III in Ref. [23], with a wedge magnet for edge focusing, a point-kick model in a rectangular bending magnet can be derived. Figure 25 shows a schematic view of the two dipole magnets with edge focusing elements, where $E_{i j}$ is the wedge magnet component and $\alpha_{i j}$ is the edge angle. In this case, the horizontal coordinate deviation due to the CSR effect after the rectangular bending dipole magnet is given by

$$
\begin{aligned}
X_{e}= & \left(\begin{array}{c}
x_{e} \\
x_{e}^{\prime}
\end{array}\right) \\
= & \left(\begin{array}{c}
\rho(1-\cos \theta) \\
\sin \theta+\tan \alpha_{2}(1-\cos \theta)
\end{array}\right) \delta \\
& +\left(\begin{array}{c}
\rho^{4 / 3}(\theta-\sin \theta) \\
\rho^{1 / 3}\left[\tan \alpha_{2}(\theta-\sin \theta)+(1-\cos \theta)\right]
\end{array}\right) k,
\end{aligned}
$$

where $x_{e}$ and $x_{e}{ }^{\prime}$ are the coordinate deviations, and $\alpha_{2}$ indicates the edge angle after the sector bending magnet. Provided that the CSR kick is applied at the center of the dipole magnet, it is described by

$$
X_{k}=\left(\begin{array}{c}
x_{k} \\
x_{k}{ }^{\prime}
\end{array}\right)=\left(\begin{array}{c}
D_{k} \\
D_{k}{ }^{\prime}
\end{array}\right) \delta+\left(\begin{array}{c}
\zeta_{k} \\
\zeta_{k}^{\prime}
\end{array}\right) k
$$

where $D_{k}$ and $D_{k}{ }^{\prime}$ are the dispersive terms of the dipole magnet, and $\zeta_{k}$ and $\zeta_{k}{ }^{\prime}$ are the CSR-induced dispersive terms.
Once the CSR kick is applied at the center of the dipole magnet and the electron beam passes through the dipole magnet, its coordinate deviations become

$$
\begin{aligned}
\left(\begin{array}{c}
x_{e} \\
x_{e}{ }^{\prime}
\end{array}\right)= & \left(\begin{array}{cc}
\cos \frac{\theta}{2} & \rho \sin \frac{\theta}{2} \\
\frac{1}{\rho}\left(\cos \frac{\theta}{2} \tan \alpha_{2}-\sin \frac{\theta}{2}\right) & \cos \frac{\theta}{2}+\sin \frac{\theta}{2} \tan \alpha_{2}
\end{array}\right) \\
& \times\left(\begin{array}{c}
x_{k} \\
x_{k}{ }^{\prime}
\end{array}\right),
\end{aligned}
$$

where the $2 \times 2$ matrix is the next half of the rectangular bending magnet in the horizontal plane. Substituting Eqs. (A1), (A2) into Eq. (A3), $D_{k}, D_{k}{ }^{\prime}, \zeta_{k}$, and $\zeta_{k}{ }^{\prime}$ can be determined.

$$
\begin{aligned}
D_{k} & =0, \\
D_{k}{ }^{\prime} & =2 \sin \frac{\theta}{2}, \\
\zeta_{k} & =\rho^{4 / 3}\left(\theta \cos \frac{\theta}{2}-2 \sin \frac{\theta}{2}\right), \\
\zeta_{k}{ }^{\prime} & =\rho^{1 / 3} \theta \sin \frac{\theta}{2} .
\end{aligned}
$$

Then, the point-kick model becomes

$$
X_{k}=\left(\begin{array}{c}
x_{k} \\
x_{k}{ }^{\prime}
\end{array}\right)=\left(\begin{array}{c}
\rho^{4 / 3} k\left(\theta \cos \frac{\theta}{2}-2 \sin \frac{\theta}{2}\right) \\
\sin \frac{\theta}{2}\left(2 \delta+\rho^{1 / 3} \theta k\right)
\end{array}\right),
$$

which is identical to that reported in Ref. [23]. Therefore, the CSR suppression condition in the rectangular bending magnet is identical to the case of a sector bending magnet; the edge focusing of the bending dipole magnet does not contribute to determining the suppression condition.

\section{APPENDIX B: CONCATENATED COMPONENTS OF THE PHASE SPACE ONLY RELATED TO $\delta_{\mathrm{CSR}}$}

In Eqs. (7), (8), many terms arise from the concatenation of the transfer map. Because each term accumulates a number of matrix components during the propagation, we check the terms $M_{15,0001}$ and $M_{15,0001}^{\prime}$ for the CSR-induced phase-space distortion as an example. These terms are listed in Table IX. 
TABLE IX. Concatenation map to obtain $M_{15,0001}$ and $M_{15,0001}^{\prime}$ components, which are related to $\delta_{\mathrm{CSR}}$. For notational simplicity, the subscript 0001 is omitted throughout the table.

\begin{tabular}{lll}
\hline \hline Number & \multicolumn{1}{c}{$\Delta x$} & \multicolumn{1}{c}{$\Delta x^{\prime}$} \\
\hline 1 & $M_{01}=0$ & $M_{01}^{\prime}=0$ \\
2 & $M_{02}=B_{16}^{(12)}$ & $M_{02}^{\prime}=B_{26}^{(12)}$ \\
3 & $M_{03}=M_{02}+M_{02}^{\prime} D_{1}$ & $M_{03}^{\prime}=M_{02}^{\prime}$ \\
4 & $M_{04}=M_{03} Q_{11}^{(1)}+M_{03}^{\prime} Q_{12}^{(1)}$ & $M_{04}^{\prime}=M_{03} Q_{21}^{(1)}+M_{03}^{\prime} Q_{22}^{(1)}$ \\
5 & $M_{05}=M_{04}+M_{04}^{\prime} D_{2}$ & $M_{05}^{\prime}=M_{04}^{\prime}$ \\
6 & $M_{06}=M_{05} Q_{11}^{(2)}+M_{05}^{\prime} Q_{12}^{(2)}$ & $M_{06}^{\prime}=M_{05} Q_{21}^{(2)}+M_{05}^{\prime} Q_{22}^{(2)}$ \\
7 & $M_{07}=M_{06}+M_{06}^{\prime} D_{2}$ & $M_{07}^{\prime}=M_{06}^{\prime}$ \\
8 & $M_{08}=M_{07} Q_{11}^{(3)}+M_{07}^{\prime} Q_{12}^{(3)}$ & $M_{08}^{\prime}=M_{07} Q_{21}^{(3)}+M_{07}^{\prime} Q_{22}^{(3)}$ \\
9 & $M_{09}=M_{08}+M_{08}^{\prime} D_{2}$ & $M_{09}^{\prime}=M_{08}^{\prime}$ \\
10 & $M_{10}=M_{09} Q_{11}^{(2)}+M_{09}^{\prime} Q_{12}^{(2)}$ & $M_{10}^{\prime}=M_{09} Q_{21}^{(2)}+M_{09}^{\prime} Q_{22}^{(2)}$ \\
11 & $M_{11}=M_{10}+M_{10}^{\prime} D_{2}$ & $M_{11}^{\prime}=M_{10}^{\prime}$ \\
12 & $M_{12}=M_{11} Q_{11}^{(1)}+M_{11}^{\prime} Q_{12}^{(1)}$ & $M_{12}^{\prime}=M_{11} Q_{21}^{(1)}+M_{11}^{\prime} Q_{22}^{(1)}$ \\
13 & $M_{13}=M_{12}+M_{12}^{\prime} D_{1}$ & $M_{13}^{\prime}=M_{12}^{\prime}$ \\
14 & $M_{14}=M_{13} B_{11}^{(21)}+M_{13}^{\prime} B_{12}^{(21)}+2 B_{16}^{(21)}$ & $M_{14}^{\prime}=M_{13} B_{21}^{(21)}+M_{13}^{\prime} B_{22}^{(21)}+2 B_{26}^{(21)}$ \\
15 & $M_{15}=M_{14}^{(22)} B_{11}^{(22)}+M_{14}^{\prime} B_{12}^{(22)}+3 B_{16}^{(22)}$ & $M_{15}^{\prime}=M_{14} B_{21}^{(22)}+M_{14}^{\prime} B_{22}^{(22)}+3 B_{26}^{(22)}$ \\
\hline \hline
\end{tabular}

For instance, $B_{16}^{(12)}$ and $B_{26}^{(12)}$ are the $R_{16}$ and $R_{26}$ components of the second half of the first dipole magnet, $D_{1}$ is the length of the first drift space, and $Q_{11}^{(3)}$ is the $R_{11}$ component of the third quadrupole magnet. Each element in the achromatic dog-leg is shown in Fig. 11.

[1] T. Shintake, H. Tanaka, T. Hara, T. Tanaka, K. Togawa, M. Yabashi, Y. Otake, Y. Asano, T. Bizen, T. Fukui et al., A compact free-electron laser for generating coherent radiation in the extreme ultraviolet region, Nat. Photonics 2, 555 (2008).

[2] H.-S. Kang, C.-K. Min, H. Heo, C. Kim, H. Yang, G. Kim, I. Nam, S. Y. Baek, H.-J. Choi, G. Mun et al., Hard X-ray free-electron laser with femtosecond-scale timing jitter, Nat. Photonics 11, 708 (2017).

[3] I. Wilson, The compact linear collider CLIC, Phys. Rep. 403, 365 (2004).

[4] E. Adli, J.-P. Delahaye, S. J. Gessner, M. J. Hogan, T. Raubenheimer, W. An, C. Joshi, and W. Mori, A beam driven plasma-wakefield linear collider: From Higgs factory to Multi-Tev, arXiv:1308.1145.

[5] E. L. Saldin, E. A. Schneidmiller, and M. Yurkov, On the coherent radiation of an electron bunch moving in an arc of a circle, Nucl. Instrum. Methods Phys. Res., Sect. A 398, 373 (1997).

[6] H. H. Braun, R. Corsini, L. Groening, F. Zhou, A. Kabel, T. Raubenheimer, R. Li, and T. Limberg, Emittance growth and energy loss due to coherent synchrotron radiation in a bunch compressor, Phys. Rev. Accel. Beams 3, 124402 (2000).

[7] M. Borland, Simple method for particle tracking with coherent synchrotron radiation, Phys. Rev. Accel. Beams 4, 070701 (2001).
[8] Y. Ding, A. Brachmann, F.-J. Decker, D. Dowell, P. Emma, J. Frisch, S. Gilevich, G. Hays, P. Hering, Z. Huang et al., Measurements and Simulations of Ultralow Emittance and Ultrashort Electron Beams in the Linac Coherent Light Source, Phys. Rev. Lett. 102, 254801 (2009).

[9] S. Bettoni, M. Aiba, B. Beutner, M. Pedrozzi, E. Prat, S. Reiche, and T. Schietinger, Preservation of low slice emittance in bunch compressors, Phys. Rev. Accel. Beams 19, 034402 (2016).

[10] Y. Ding, K. L. F. Bane, W. Colocho, F. J. Decker, P. Emma, J. Frisch, M. W. Guetg, Z. Huang, R. Iverson, J. Krzywinski et al., Beam shaping to improve the free-electron laser performance at the linac coherent light source, Phys. Rev. Accel. Beams 19, 100703 (2016).

[11] T. Charles, D. Paganin, A. Latina, M. Boland, and R. Dowd, Current-horn suppression for reduced coherentsynchrotron-radiation-induced emittance growth in strong bunch compression, Phys. Rev. Accel. Beams 20, 030705 (2017).

[12] P. Muggli, B. E. Blue, C. E. Clayton, S. Deng, F. J. Decker, M. J. Hogan, C. Huang, R. Iverson, C. Joshi, T. C. Katsouleas et al., Meter-Scale Plasma-Wakefield Accelerator Driven by a Matched Electron Beam, Phys. Rev. Lett. 93, 014802 (2004).

[13] R. Gholizadeh, T. Katsouleas, P. Muggli, C. Huang, and W. Mori, Preservation of Beam Emittance in the Presence of Ion Motion in Future High-Energy PlasmaWakefield-Based Colliders, Phys. Rev. Lett. 104, 155001 (2010).

[14] T. Mehrling, J. Grebenyuk, F. Tsung, K. Floettmann, and J. Osterhoff, Transverse emittance growth in staged laserwakefield acceleration, Phys. Rev. Accel. Beams 15, 111303 (2012).

[15] V. Yakimenko, M. Fedurin, V. Litvinenko, A. Fedotov, D. Kayran, and P. Muggli, Experimental Observation of Suppression of Coherent-Synchrotron-Radiation-Induced 
Beam-Energy Spread with Shielding Plates, Phys. Rev. Lett. 109, 164802 (2012).

[16] G. Ha, M. Conde, D. Doran, J. Power et al., Limiting effects in the double EEX beamline, in 8th Int. Particle Accelerator Conf.(IPAC'17), Copenhagen, Denmark, 14â 19 May, 2017 (JACOW, Geneva, Switzerland, 2017), pp. 3858-3861.

[17] C. Mitchell, J. Qiang, and P. Emma, Longitudinal pulse shaping for the suppression of coherent synchrotron radiation-induced emittance growth, Phys. Rev. Accel. Beams 16, 060703 (2013).

[18] D. Douglas, Suppression \& Enhancement of CSR-driven Emittance Degradation in the IR-FEL Driver, Technical Report JLAB-TN-98-012, 1998.

[19] D. Douglas, S. Benson, A. Hutton, G. Krafft, R. Li, G. Neil, Y. Roblin, C. Tennant, and C.-Y. Tsai, Control of coherent synchrotron radiation and micro-bunching effects during transport of high brightness electron beams, arXiv: 1403.2318.

[20] R. Hajima, A first-order matrix approach to the analysis of electron beam emittance growth caused by coherent synchrotron radiation, Jpn. J. Appl. Phys. 42, L974 (2003).

[21] R. Hajima, Emittance compensation in a return arc of an energy-recovery linac, in Free Electron Lasers 2003 (Elsevier, New York, 2004), pp. 335-339.

[22] S. Di Mitri, M. Cornacchia, and S. Spampinati, Cancellation of Coherent Synchrotron Radiation Kicks with Optics Balance, Phys. Rev. Lett. 110, 014801 (2013).

[23] Y. Jiao, X. Cui, X. Huang, and G. Xu, Generic conditions for suppressing the coherent synchrotron radiation induced emittance growth in a two-dipole achromat, Phys. Rev. Accel. Beams 17, 060701 (2014).

[24] X.-Y. Huang, Y. Jiao, G. Xu, and X.-H. Cui, Suppression of the emittance growth induced by coherent synchrotron radiation in triple-bend achromats, Chin. Phys. C 39, 057001 (2015).

[25] M. Venturini, Design of a triple-bend isochronous achromat with minimum coherent-synchrotron-radiationinduced emittance growth, Phys. Rev. Accel. Beams 19, 064401 (2016).

[26] A. Caldwell, K. Lotov, A. Pukhov, and F. Simon, Protondriven plasma-wakefield acceleration, Nat. Phys. 5, 363 (2009).

[27] E. Gschwendtner, E. Adli, L. Amorim, R. Apsimon, R. Assmann, A.-M. Bachmann, F. Batsch, J. Bauche, V. B. Olsen, M. Bernardini et al., AWAKE, the advanced proton driven plasma wakefield acceleration experiment at CERN, Nucl. Instrum. Methods Phys. Res., Sect. A 829, 76 (2016).

[28] E. Adli, A. Ahuja, O. Apsimon, R. Apsimon, A.-M. Bachmann, D. Barrientos, F. Batsch, J. Bauche, V. B. Olsen, M. Bernardini et al., Acceleration of electrons in the plasma wakefield of a proton bunch, Nature (London) 561, 363 (2018).

[29] P. Muggli, Physics to plan AWAKE Run 2, arXiv: 1911.07534.

[30] M. Turner, E. Adli, A. Ahuja, O. Apsimon, R. Apsimon, A.-M. Bachmann, M. B. Marin, D. Barrientos, F. Batsch, J. Batkiewicz et al., Experimental Observation of Plasma Wakefield Growth Driven by the Seeded
Self-Modulation of a Proton Bunch, Phys. Rev. Lett. 122, 054801 (2019).

[31] E. Adli, Towards AWAKE applications: Electron beam acceleration in a proton driven plasma wake, in 7th Int. Particle Accelerator Conf.(IPAC'16), Busan, Korea, May 8, 13, 2016 (JACoW, Geneva, 2016), pp. 2557-260.

[32] M. Borland, Elegant: A flexible SDDS-compliant code for accelerator simulation, Tech. Rep. (Argonne National Lab., IL (US), 2000).

[33] R. England, J. Rosenzweig, G. Andonian, P. Musumeci, G. Travish, and R. Yoder, Sextupole correction of the longitudinal transport of relativistic beams in dispersionless translating sections, Phys. Rev. Accel. Beams 8, 012801 (2005).

[34] K. L. Brown, First-and Second-order matrix theory for the design of beam transport systems and charged particle spectrometers, Tech. Rep. (Stanford Linear Accelerator Center, CA., 1971).

[35] B. W. S. L. Montague, Linear optics for improved chromaticity correction, Tech. Report No. SCAN-0009131, 1979.

[36] C. A. Lindstrøm and E. Adli, Design of general apochromatic drift-quadrupole beam lines, Phys. Rev. Accel. Beams 19, 071002 (2016).

[37] Mad-methodical accelerator design, http://mad.web.cern $. \mathrm{ch} / \mathrm{mad} /$.

[38] M. Conte and W. W. MacKay, Introduction To The Physics Of Particle Accelerators (World Scientific Publishing Company, Singapore, 2008).

[39] Y. Cai, Symplectic maps and chromatic optics in particle accelerators, Nucl. Instrum. Methods Phys. Res., Sect. A 797, 172 (2015).

[40] H. Wiedemann, Particle Accelerator Physics (Springer Nature, New York, 2015).

[41] J. Lim, P. Frigola, G. Travish, J. Rosenzweig, S. Anderson, W. Brown, J. Jacob, C. Robbins, and A. Tremaine, Adjustable, short focal length permanent-magnet quadrupole based electron beam final focus system, Phys. Rev. Accel. Beams 8, 072401 (2005).

[42] P. Muggli, Beam-driven, plasma-based particle accelerators, arXiv:1705.10537.

[43] V. K. B. Olsen, E. Adli, and P. Muggli, Emittance preservation of an electron beam in a loaded quasilinear plasma wakefield, Phys. Rev. Accel. Beams 21, 011301 (2018).

[44] R. Lehe, M. Kirchen, I. A. Andriyash, B. B. Godfrey, and J.-L. Vay, A spectral, quasi-cylindrical and dispersion-free Particle-In-Cell algorithm, Comput. Phys. Commun. 203, 66 (2016).

[45] N. Barov, J. Rosenzweig, M. Conde, W. Gai, and J. Power, Observation of plasma wakefield acceleration in the underdense regime, Phys. Rev. Accel. Beams 3, 011301 (2000).

[46] W. An, M. Zhou, N. Vafaei-Najafabadi, K. Marsh, C. Clayton, C. Joshi, W. Mori, W. Lu, E. Adli, S. Corde et al., Strategies for mitigating the ionization-induced beam head erosion problem in an electron-beam-driven plasma wakefield accelerator, Phys. Rev. Accel. Beams 16, 101301 (2013).

[47] X. L. Xu, J. F. Hua, F. Li, C. J. Zhang, L. X. Yan, Y. C. Du, W. H. Huang, H. B. Chen, C. X. Tang, W. Lu et al., 
Phase-space Dynamics of Ionization Injection in Plasma-based Accelerators, Phys. Rev. Lett. 112, 035003 (2014).

[48] A. Aschikhin, T. J. Mehrling, A. M. de la Ossa, and J. Osterhoff, Analytical model for the uncorrelated emittance evolution of externally injected beams in plasma-based accelerators, Nucl. Instrum. Methods Phys. Res., Sect. A 909, 414 (2018).
[49] A. Khachatryan, A. Irman, F. Van Goor, and K.-J. Boller, Femtosecond electron-bunch dynamics in laser wakefields and vacuum, Phys. Rev. Accel. Beams 10, 121301 (2007).

[50] P. Michel, C. B. Schroeder, B. A. Shadwick, E. Esarey, and W. P. Leemans, Radiative damping and electron beam dynamics in plasma-based accelerators, Phys. Rev. E 74, 026501 (2006). 